\title{
Ensemble des troubles causés par l'alcoolisation foetale : lignes directrices canadiennes concernant le diagnostic
}

\author{
Albert E. Chudley, Julianne Conry, Jocelynn L. Cook, Christine Loock, Ted Rosales, \\ Nicole LeBlanc
}

Résumé

LE DIAGNOSTIC DE L'ENSEMBLE DES TROUBLES CAUSÉS PAR L'ALCOOLISATION FOETALE (ETCAF) est complexe et l'élaboration de lignes directrices concernant le diagnostic est justifiée. Un sous-comité du Comité consultatif national de l'Agence de santé publique du Canada sur I'Ensemble des troubles causés par l'alcoolisation fœetale a examiné, analysé et intégré les méthodes de diagnostic actuelles afin de parvenir à une méthode de diagnostic normalisée faisant le consensus au Canada. L'objet du présent document est d'examiner et de clarifier l'utilisation des systèmes de diagnostic actuels et de formuler des recommandations quant à leur application pour le diagnostic des déficiences liées à l'ETCAF chez des individus de tous les âges. Les lignes directrices sont fondées sur un vaste consensus de praticiens et d'autres intervenants spécialisés dans le domaine. Ces lignes directrices ont été organisées en sept catégories, soit le dépistage et l'orientation vers les spécialistes, l'examen physique et le diagnostic différentiel, l'évaluation du comportement neurologique, le traitement et le suivi, les antécédents de consommation d'alcool de la mère pendant la grossesse, les critères du diagnostic pour le syndrome d'alcoolisation fotale (SAF), SAF partiel et troubles neurologiques du développement liés à l'alcool, I'harmonisation de I'Institute of Medicine (IOM) et des approches du Code diagnostique à 4 chiffres. Le diagnostic exige une évaluation complète des antécédents, ainsi qu'un examen physique et du comportement neurologique, tout en recourant à une approche multidisciplinaire. Les présentes lignes directrices pour le diagnostic du syndrome d'alcoolisation fœetale et des déficiences qui y sont associées sont les premières à avoir été élaborées au Canada et elles sont fondées sur la consultation d'un grand éventail de spécialistes du diagnostic.

$\mathrm{D}$ ans le présent document, nous traitons de la méthode du diagnostic des déficiences liées à l'exposition prénatale à l'alcool. L'ensemble des troubles causés par l'alcoolisation foetale (ETCAF) et sa forme la plus visible, le syndrome d'alcoolisation foetale (SAF), représentent une sérieuse préoccupation pour les Canadiens et les Canadiennes sur les plans sanitaire et social. L'ETCAF est un terme générique employé pour décrire la gamme des problèmes qui peuvent survenir chez un individu dont la mère consommait de l'alcool au cours de sa grossesse. Ces problèmes peuvent inclure des déficiences physiques ou intellectuelles ainsi que des déficiences au niveau du comportement et de l'apprentissage avec des séquelles permanentes. L'expression ETCAF n'est pas proposée en vue de son emploi comme diagnostic clinique.

L'ETCAF est attribuable à la consommation d'alcool par la mère pendant la grossesse et a des répercussions sur la personne atteinte, sa mère, sa famille et sa communauté. Depuis que ces troubles ont été décrits pour la première fois en $1973^{1}$, il est apparu qu'ils sont complexes et que les personnes atteintes en présentent une grande gamme d'expressions allant du grave retard de croissance, de la déficience intel- lectuelle, des anomalies congénitales et des dysmorphies faciales caractéristiques, jusqu'à une croissance normale, des capacités intellectuelles et des traits faciaux normaux mais avec des déficiences permanentes au niveau de plusieurs fonctions du cerveau. Ces troubles exigent un diagnostic médical dans le contexte d'une évaluation multidisciplinaire. L'ETCAF n'est pas en soi un terme diagnostique. L'objet du présent document est d'analyser et de clarifier l'utilisation des systèmes de diagnostic actuels et de formuler des recommandations quant à leur application pour le diagnostic des déficiences liées à l'ETCAF chez des individus de tous les âges. Pour une description des caractéristiques de l'ETCAF et son évolution naturelle, les lecteurs sont priés de consulter certaines des analyses plus générales ${ }^{2-7}$.

\section{Épidémiologie de l'ETCAF}

Aux États-Unis, la prévalence du SAF serait de 1 à 3 pour 1000 naissances vivantes et celle de l'ETCAF, de 9,1 pour 1000 naissances vivantes ${ }^{8-11}$. Toutefois, il peut souvent arriver que le diagnostic soit retardé ou entièrement manqué

Il n'existe pas de statistiques nationales sur le taux de l'ETCAF au Canada, bien que certaines études aient estimé la prévalence dans de petites populations.

Dans une collectivité autochtone isolée de la ColombieBritannique, on a constaté un taux de prévalence de l'ETCAF de 190 pour 1000 naissances vivantes ${ }^{12}$, et dans le Nord-Est du Manitoba, une incidence de 7,2 pour 1000 naissances vivantes $^{13}$. Une autre étude menée au Manitoba dans une collectivité des Premières nations ${ }^{14}$ a révélé une prévalence du SAF et du SAF partiel de 55 à 101 pour 1000 naissances. Dans leur étude, Asante et Nelms-Matzke ${ }^{15}$ ont estimé le taux du SAF et de ses effets connexes à 46 pour 1000 enfants autochtones canadiens dans le Yukon et à 25 pour 1000 dans le Nord de la Colombie-Britannique. En se fondant sur les cas ayant été dirigés vers une clinique de diagnostic en Saskatchewan, le taux du SAF est estimé à 0,589 pour 1000 naissances vivantes pour la période de 1988 à 1992 et à 0,515 pour 1000 de 1973 à $1977^{16}$. Il convient toutefois de ne pas généraliser et de ne pas tirer de conclusions de ces données en ce qui concerne d'autres communautés, d'autres populations ou la population canadienne en général.

\section{Facteurs de risque}

La perception commune voulant que l'ETCAF soit associé à un contexte ethno-culturel particulier est fausse. Toutefois, les données suggèrent que les facteurs de risque associés à l'exposition prénatale à l'alcool comportent notamment un âge plus élevé et un niveau d'éducation moindre de la mère, 
une exposition prénatale à la cocaïne et à la cigarette, des changements dans la garde de l'enfant, un statut socioéconomique inférieur et une consommation d'alcool et d'autres drogues par le père pendant la grossesse ${ }^{17}$, et l'accès réduit aux soins et aux services prénatals et postnatals, une nutrition inadéquate et un milieu peu propice au développement (p. ex., le stress, les mauvais traitements, la négligence) ${ }^{18}$.

Lors d'une étude de suivi de cinq ans visant les mères naturelles d'enfants atteints de SAF complet, Astley et ses collaborateurs $^{19}$ ont trouvé que ces femmes avaient des antécédents raciaux, scolaires et économiques variés. Elles éprouvaient souvent des troubles de santé mentale non traités ou insuffisamment traités, souffraient d'isolement social, étaient victimes de mauvais traitements et avaient été victimes de graves sévices sexuels pendant leur enfance.

Puisqu'il n'existe aucune étude à grande échelle sur les facteurs de risque, et que les risques sont interreliés et pourraient varier pour des populations différentes, il est difficile de présenter des chiffres précis sur le risque relatif. Cependant, le facteur de risque le plus important de l'ETCAF est lié au taux élevé d'alcool dans le sang, au moment de l'exposition pendant le développement du foetus, aux habitudes de consommation, p. ex., la consommation épisodique excessive (quatre consommations ou plus par épisode) et la fréquence de la consommation. Bien qu'il ne semble pas y avoir de seuil d'exposition, les effets semblent liés au rapport dose-réponse ${ }^{17,20,21}$.

\section{Importance d'un diagnostic précoce}

Il est essentiel de poser un diagnostic précoce afin d'assurer l'accès aux interventions et aux ressources qui pourraient atténuer le développement des «incapacités secondaires» subséquentes (p. ex., le chômage, les problèmes de santé mentale, les démêlés avec la justice, les comportements sexuels inappropriés, les perturbations dans la scolarité) chez les personnes atteintes ${ }^{22}$. De plus, un diagnostic précoce permettra aussi une intervention, un counselling et un traitement appropriés pour la mère et pourrait éviter la naissance d'autres enfants atteints de $\mathrm{SAF}^{23}$. En outre, les fournisseurs de soins pourraient être incités à obtenir un diagnostic et un appui pour des frères ou sœurs qui n'ont jamais été évalués. On trouvera dans d'autres sources des études sur le suivi médical et la gestion du comportement de personnes atteintes par l'ETCAF ${ }^{3,24}$. Astley et Clarren ${ }^{25}$ suggèrent qu'un diagnostic précis et opportun est essentiel à l'amélioration des résultats, puisqu'un diagnostic erroné résulte en des soins mal adaptés au patient, un risque accru d'incapacités secondaires et des occasions ratées de prévention, et mène à des estimations inexactes de l'incidence et de la prévalence. Ensemble, ces inexactitudes pourraient entraver les efforts visant à assurer des services sociaux et des soins de santé adéquats aux populations vulnérables et empêcher une évaluation précise des interventions de prévention primaire.

Étant donné que la capacité et l'expertise sont limitées et que l'évaluation exige la participation de plusieurs profes- sionnels à une évaluation diagnostique multidisciplinaire complète, un diagnostic n'est actuellement établi que pour une fraction des personnes atteintes. Les résultats ${ }^{26} \mathrm{~d}^{\prime} u n e$ enquête pancanadienne sur les connaissances et les attitudes des professionnels de la santé suggèrent que les praticiens ont besoin de lignes directrices normalisées en matière de diagnostic et de plus d'éducation et de formation professionnelles pour pouvoir établir un diagnostic. Compte tenu de ces préoccupations, le Comité consultatif national de Santé Canada sur l'ETCAF, ainsi que les spécialistes et les praticiens du diagnostic et du traitement du SAF, présentent les lignes directrices suivantes pour le diagnostic.

\section{Processus de l'élaboration des lignes directrices}

Les présentes lignes directrices sont le résultat de plus de 10 consultations en personne avec des spécialistes canadiens et américains du diagnostic du SAF et des incapacités qui y sont associées (Annexe 1). Bon nombre des participants offrent actuellement des services de diagnostic un peu partout au Canada. Un groupe diversifié de spécialistes et d'organisations et sociétés professionnelles, ainsi que les gouvernements fédéral, provinciaux et territoriaux, ont examiné les lignes directrices et fourni une rétroaction. Des lignes directrices sont présentées dans six domaines liés au processus de diagnostic : 1 . le dépistage et l'orientation vers les spécialistes; 2. l'examen physique et le diagnostic différentiel; 3. l'évaluation du comportement neurologique; 4. le traitement et le suivi; 5. les antécédents de consommation d'alcool par la mère pendant la grossesse; 6 . les critères de diagnostic du SAF, du SAF partiel et des troubles neurologiques du développement liés à l'alcool. Nous incluons également des recommandations pour mieux harmoniser les deux principales approches de diagnostic.

Il existe de nombreuses méthodes de diagnostic et le groupe de travail a cherché à les intégrer afin d'assurer des diagnostics uniformes partout au Canada. Nos connaissances actuelles sur la complexité des incapacités associées à l'exposition prénatale à l'alcool indiquent qu'une évaluation multidisciplinaire complète est requise pour pouvoir établir un dia-gnostic précis et formuler des recommandations de traitement. Nous recommandons une telle méthode multidisciplinaire. Cette méthode permettra également de recueillir des données canadiennes afin d'estimer l'incidence et la prévalence de l'ETCAF. Ces renseignements sont essentiels pour connaître les programmes et services de prévention et d'intervention requis et les élaborer.

\section{Historique et terminologie du diagnostic du SAF}

On attribue à Lemoine et ses collaborateurs d'avoir reconnu pour la première fois un type d'anomalies congénitales et de troubles du développement chez les enfants nés de parents alcooliques ${ }^{27}$. Un ensemble précis d'anomalies congénitales attribuées à la consommation d'alcool par la 
mère a été décrit aux États-Unis ${ }^{1,28}$. Cet ensemble d'anomalies, appelé le SAF, consiste en anomalies faciales (l'aplatissement du sillon naso-labial [l'espace entre la lèvre supérieure et le nez], l'amincissement du vermillon [la partie rouge exposée à la vue de la lèvre supérieure], le raccourcissement des fentes palpébrales), retards de croissance prénatals ou postnatals ou les deux, et troubles du système nerveux central et du comportement neurologique. L'alcool agit probablement par le biais de nombreux mécanismes, et une gamme d'incapacités ont été observées en l'absence de dysmorphies reflétant les divers degrés d'affectation pendant le développement fœetal; sans aucun doute, le moment et le degré de l'exposition sont des variables importantes contribuant à la variation. Ainsi, l'expression «Effets soupçonnés de l'alcool sur le foetus» (EAF) a été créée ${ }^{29}$. Le profil de ces «effets» a été raffiné par la suite par l'Institute of Medicine américain, lequel a publié en 1996 des recommandations pour le diagnostic du SAF en consultation avec un comité de spécialistest. Les catégories de diagnostic décrites sont, notamment : le SAF avec et sans exposition à l'alcool confirmée, le SAF partiel (SAFp), les anomalies congénitales liées à l'alcool (ACLA) et les troubles neurologiques du développement liés à l'alcool (TNDLA) (Tableau 1).

Vers la fin des années 1990, une autre stratégie de diagnostic a été élaborée par Astley et Clarren ${ }^{25,30}$. Ils ont créé un Code diagnostique à 4 chiffres en utilisant les données d'un réseau de cliniques, le Washington State Fetal Alcohol Syndrome Diagnostic and Prevention Network. Leur système de diagnostic utilise des échelles de mesures quantitatives objectives et des définitions des cas particuliers. Les quatre chiffres du code traduisent l'importance de l'expression des quatre principales caractéristiques diagnostiques du SAF dans l'ordre suivant : retard de croissance, phénotype facial du SAF, endommagement ou dysfonctions du système nerveux central, exposition à l'alcool pendant la grossesse. L'importance de l'expression de chaque caractéristique est cotée indépendamment sur une échelle de Likert à quatre points, le chiffre 1 indiquant l'absence totale de la caractéristique et le chiffre 4 indiquant son expression extrême. Le Code diagnostique à 4 chiffres est maintenant utilisé pour le diagnostic, le dépistage et le suivi dans des cliniques de toutes les régions des ÉtatsUnis et du Canada. Cet article utilise la terminologie de l'édition 2004 du Code diagnostique à 4 chiffres d'Astley.*

Bien que les méthodes employées diffèrent, les critères fondamentaux de l'IOM et du Code diagnostique à 4 chiffres sont semblables. Certaines cliniques choisissent d'intégrer les outils de diagnostic et la précision offerts par le Code diagnostique à 4 chiffres, en utilisant les catégories de diagnostic et la terminologie recommandées par le comité de l'IOM. Bien qu'on ait publié les critères de l'IOM et le Code diagnostique à 4 chiffres, de nombreux cliniciens continuent

*Astley SJ. Diagnostic Guide for Fetal Alcohol Spectrum Disorders: The 4-Digit Diagnostic Code (3 édition). Seattle : University of Washington Publication Services; 2004. d'utiliser la méthode gestaltiste, moins souhaitable et possiblement trompeuse (Tableau 2).

\section{Le processus de diagnostic}

Le processus de diagnostic se compose du dépistage et de l'orientation vers les spécialistes, de l'examen physique et du diagnostic différentiel, de l'évaluation du comportement neurologique, et du traitement et suivi.

Étant donné la complexité et la gamme d'expression des dysfonctions liées à l'exposition prénatale à l'alcool, une équipe multidisciplinaire est essentielle pour poser un diagnostic précis et complet et formuler des recommandations de traitement. Le processus d'évaluation débute par la reconnaissance du besoin d'un diagnostic et se termine avec la mise en œuvre des recommandations appropriées. L'équipe multidisciplinaire de diagnostic peut être géographique, régionale ou virtuelle; elle peut aussi accepter des patients de collectivités éloignées qui lui sont envoyés et les évaluer en utilisant la télémédecine.

L'équipe de base peut varier selon le contexte spécifique, mais idéalement elle devrait réunir les professionnels suivants possédant les compétences, la formation et l'expérience voulues dans leur discipline :

- un coordonnateur de gestion du cas (p. ex., un infirmière ou un travailleur social);

- un médecin formé expressément au diagnostic de l'ETCAF;

- un psychologue;

- un ergothérapeute;

- un orthophoniste.

Les membres supplémentaires peuvent comprendre des conseillers en toxicomanie, des travailleurs des services à l'enfance, des interprètes culturels, des travailleurs en santé mentale, des parents ou des fournisseurs de soins, des agents de probation, des psychiatres, des enseignants, des orienteurs professionnels, des infirmiers et infirmières, des généticiens ou des dysmorphologistes, des neuropsychologues, des thérapeutes familiaux, etc.

\section{Observations}

Il est clair que des fonds sont requis pour le développement, la formation et le soutien d'équipes de diagnostic multidisciplinaires afin de s'assurer que les centres importants auront l'expertise et les capacités nécessaires pour servir leurs collectivités. Pour optimiser les résultats du diagnostic, la communauté et la famille doivent être préparées, consentir à l'évaluation diagnostique et être prêtes à y participer. Le processus de diagnostic doit être sensible aux besoins de la famille et du fournisseur de soins. Dans chaque communauté, les cas référés doivent être évalués et classés par ordre de priorité. La famille et le tuteur doivent être d'accord avec le but du diagnostic. Ils doivent être sensibilisés aux conséquences psychosociales qui pourraient suivre un diagnostic de l'ETCAF (p. ex., le sentiment de culpabilité et de colère, particulièrement envers la mère naturelle, ou la stigmatisation possible de l'enfant). La famille 
ou le tuteur auront probablement besoin d'aide pour s'engager dans le processus de diagnostic avec confiance. Cette aide pourrait comprendre une certaine éducation préparatoire sur l'ETCAF et des liens avec des services de soutien et des ressources communautaires.

On devra obtenir des renseignements de diverses sources (p. ex., les dossiers scolaires, les dossiers d'hospitalisation, les services sociaux, les évaluations précédentes) incluant des rencontres avec les professionnels pertinents connaissant le patient (p. ex., des enseignants, des médecins, des travailleurs sociaux, des psychologues). D'autre documentation pertinente comprendra les dossiers sur la naissance et la grossesse, les dossiers médicaux et d'hospitalisation, les dossiers d'adoption, les dossiers scolaires, les tests de compétence, les évaluations du développement, les évaluations psychologiques et psychométriques, les rapports juridiques et les antécédents familiaux.

L'évaluation complète de l'équipe de diagnostic fournit des renseignements importants sur les besoins uniques de la personne et permet d'adapter les interventions en fonction de ses forces et de ses défis particuliers. Le rapport préparé à la suite du diagnostic devrait énoncer le fondement du diagnostic en incluant les antécédents de consommation d'alcool, les critères physiques et les données psychologiques.

Les équipes multidisciplinaires travaillent de concert avec des partenaires et des ressources communautaires pour élaborer et mettre en œuvre des plans de gestion visant à maximiser le potentiel de la personne atteinte. Après l'évaluation, un rapport contenant des recommandations devrait être produit et mis à la disposition des fournisseurs de soins et des éducateurs. Il faudrait discuter des conclusions de l'équipe avec les personnes chargées de la garde de l'enfant. Les enfants plus âgés qui sont en mesure de comprendre devraient apprendre le diagnostic directement de l'équipe. L'équipe peut également assumer la responsabilité d'assurer et de faciliter le suivi auprès de la famille et des ressources communautaires en ce qui concerne l'application des recommandations. En bout de ligne, le processus de diagnostic mènera à des recommandations de gestion concrètes qui amélioreront la vie des personnes atteintes, de leurs familles et de leurs collectivités.

Tableau 1 : Critères de diagnostic du syndrome d'alcoolisation fœetale (SAF) et des effets liés à l'alcool, selon I'Institute of Medicine ${ }^{4}$.

\section{Syndrome d'alcoolisation fotale (SAF)}

1. SAF avec confirmation de l'exposition de la mère à l'alcool*

A. Exposition de la mère à l'alcool confirmée*

B. Présence d'anomalies caractéristiques des traits faciaux tels qu'un raccourcissement des fentes palpébrales et des anomalies dans la région prémaxillaire (p. ex., minceur de la lèvre supérieure, aplatissement du sillon naso-labial, et aplatissement médiofacial)

C. Retard de croissance, au moins dans I'un des aspects suivants :

- petit poids à la naissance pour l'âge fotal

- perte de poids avec le temps, non reliée à la nutrition

- poids insuffisant par rapport à la taille

D. Anomalies neurologiques du développement du système nerveux central, au moins dans l'un des aspects suivants :

- petit périmètre crânien à la naissance

- anomalies structurales du cerveau (p. ex., microcéphalie, agénésie partielle ou complète du corps calleux, hypoplasie cérébelleuse)

- troubles neurologiques légers ou profonds (en fonction de l'âge), tels qu'une déficience de la motricité fine, la surdité neurosensorielle, une mauvaise démarche en tandem, une mauvaise coordination œil-main

2. SAF sans confirmation de l'exposition de la mère à l'alcool

$\mathrm{B}, \mathrm{C}$ et $\mathrm{D}$ comme ci-dessus

3. SAF partiel avec confirmation de l'exposition de la mère à l'alcool

A. Exposition de la mère à l'alcool confirmée*

B. Présence de certains des traits faciaux caractéristiques

Soit C, D ou E

C. Présence d'un retard de croissance, au moins dans l'un des aspects suivants :

- poids à la naissance peu élevé pour l'âge fotal

- perte de poids avec le temps, non reliée à la nutrition

- poids insuffisant par rapport à la taille

D. Présence d'anomalies neurologiques du développement du SNC, par ex.,

- petit périmètre crânien à la naissance

- anomalies structurales du cerveau (p. ex., microcéphalie, agénésie partielle ou complète du corps calleux, hypoplasie cérébelleuse)

- troubles neurologiques légers ou profonds (en fonction de l'âge), tels qu'une déficience de la motricité fine, la surdité neurosensorielle, une mauvaise démarche en tandem, une mauvaise coordination œeil-main

E. Présence d'une série complexe d'anomalies comportementales ou cognitives incompatibles avec le niveau de développement et ne pouvant être expliquées par les antécédents familiaux ou le milieu seulement, telles que des difficultés d'apprentissage; des déficiences dans le rendement scolaire; un mauvais contrôle des impulsions; des problèmes de perception sociale; des déficiences dans le langage réceptif et expressif; une capacité réduite d'abstraction ou de métacognition; des déficiences particulières en mathématiques; ou des problèmes de mémoire, d'attention ou de jugement. 
Le Canada est un grand pays et de vastes distances séparent ses collectivités, certaines étant éloignées et isolées. Les spécialistes qui offrent des consultations en région éloignée ont besoin d'une formation spéciale en évaluation de l'ETCAF et doivent travailler en collaboration avec des centres qui disposent d'équipes multidisciplinaires pour aider au processus de diagnostic. Un certain nombre d'outils peuvent être utiles pour poser un diagnostic à distance. Un plus grand recours à la télémédecine, par exemple, permettra d'évaluer des enfants dans les collectivités éloignées $^{31}$. Les photographies numériques ${ }^{32,33}$ envoyées par voie électronique à des équipes situées dans des centres plus importants et la scintigraphie de surface à trois dimensions au laser ${ }^{34,35}$ offrent aussi des possibilités.

Nous reconnaissons que la capacité, même de certaines collectivités importantes du Canada, de poser un diagnostic de SAF en utilisant une approche axée sur une équipe multidisciplinaire est actuellement limitée. Les professionnels devraient faire le meilleur usage des ressources et de l'expertise disponibles afin de fournir une évaluation et un plan de traitement précis aux personnes atteintes et leurs familles, en reconnaissant le rôle primordial de la psychologie.

\section{Le dépistage et l'orientation vers les spécialistes}

\section{Recommandations}

1.1 Toutes les femmes enceintes et celles venant d'accoucher devraient être soumises au dépistage de la consommation

\section{Tableau 1 : suite}

\section{Effets liés à l'alcool}

États cliniques avec antécédents d'exposition de la mère à l'alcool*t, et pour lesquels la recherche clinique ou sur les animaux a établi un lien entre l'ingestion d'alcool par la mère et un résultat observé. Il existe deux catégories d'effets liés à l'alcool, qui peuvent toutes deux être en jeu; dans ce cas, il faut rendre les deux diagnostics.

4. Anomalies congénitales liées à l'alcool (ACLA)

Liste des anomalies congénitales, y compris les malformations et dysplasies

Cardiaques

Communication interauriculaire

Communication interventriculaire

Squelettales

Hypoplasie des ongles

Auriculaires raccourcis

Synostose radiocubitale

Difformités en flexion

Camptodactylie

Rénales

Reins aplasiques, reins dysplasiques

Reins hypoplastiques

Reins en forme de fer à cheval

Oculaires

Strabisme

Anomalies des vaisseaux sanguins rétiniens

Auditives

Surdité de transmission

Autres

Presque toutes les malformations ont été décrites chez des patients atteints du SAF. L'étiologie de la plupart de ces anomalies à une tératogenèse causée par l'alcool reste incertaine.

5. Troubles neurologiques du développement liés à l'alcool (TNDLA)

Présence de $\mathrm{A}$ ou $\mathrm{B}$ ou les deux.

A. Anomalies neurologiques du développement du SNC prenant n'importe lequel des aspects suivants :

- petit périmètre crânien à la naissance

- anomalies structurales du cerveau (p. ex., microcéphalie, agénésie partielle ou complète du corps calleux, hypoplasie cérébelleuse)

- troubles neurologiques légers ou profonds (en fonction de l'âge), tels qu'une déficience de la motricité fine, la surdité neurosensorielle, une mauvaise démarche en tandem, une mauvaise coordination œil-main

B. Présence d'une série complexe d'anomalies comportementales ou cognitives incompatibles avec le niveau de développement et ne pouvant être expliquées par les antécédents familiaux ou le milieu seulement, telles que des difficultés d'apprentissage; des déficiences dans le rendement scolaire; un mauvais contrôle des impulsions; des problèmes de perception sociale; des déficiences dans le langage réceptif et expressif; une capacité réduite d'abstraction ou de métacognition; des déficiences particulières en mathématiques; ou des problèmes de mémoire, d'attention, ou de jugement.

\footnotetext{
* Des habitudes de consommation excessive caractérisées par une consommation régulière importante ou par des épisodes de consommation excessive. Ces habitudes peuvent se manifester par des épisodes fréquents d'intoxication, un développement de la tolérance ou de symptômes de sevrage, des problèmes sociaux liés à la consommation d'alcool, des problèmes judiciaires liés à la consommation d'alcool, un comportement physiquement dangereux lors de la consommation d'alcool, ou des problèmes médicaux liés à l'alcool tels que les maladies du foie.

† À mesure que d'autres recherches seront effectuées et que, le cas échéant, des quantités d'alcool moins importantes ou d'autres habitudes de consommation seront associées à des ACLA ou à des TNDLA, ces habitudes de consommation d'alcool devraient être incorporées dans les critères de diagnostic.
} 
d'alcool avec des outils de dépistage validés (p. ex., le TACE, le TWEAK) administrés par les fournisseurs de soins de santé pertinents. Les femmes à risque de consommer beaucoup d'alcool devraient recevoir une brève intervention précoce (par ex., du counselling).

1.2 L'abstinence devrait être recommandée à toutes les femmes pendant la grossesse, étant donné que la consommation continue d'alcool par la mère pendant sa grossesse risque de soumettre le fotus aux effets liés à l'exposition prénatale à l'alcool.

1.3 Il faudrait diriger les personnes vers des spécialistes pour un diagnostic possible de l'ETCAF dans les situations suivantes :

a. présence de trois traits faciaux caractéristiques (les fentes palpébrales raccourcies, le sillon naso-labial lisse ou aplati, le vermillon de la lèvre aminci);

b. preuve d'une exposition prénatale à l'alcool importante à un niveau que l'on sait associé à des effets physiques ou de développement, ou les deux;

c. présence d'un trait facial caractéristique ou plus et de retards de croissance, plus exposition prénatale à l'alcool importante confirmée ou probable;

d. présence d'un trait facial caractéristique ou plus et d'un trouble du système nerveux central ou plus, plus exposition prénatale à l'alcool importante confirmée ou probable;

e. présence d'un trait facial caractéristique ou plus et de retards de croissance prénatals ou postnatals, ou les deux (égal ou inférieur au 10e percentile [écart-type de 1,5 au-dessous de la moyenne]) et d'un trouble du système nerveux central ou plus, plus exposition prénatale à l'alcool importante confirmée ou probable.

1.4 Les personnes qui ont des difficultés d'apprentissage ou des troubles de comportement, ou les deux, mais n'ont pas de traits physiques caractéristiques ou de dysmorphies et n'ont pas d'histoire connue ou probable d'exposition à l'alcool devraient être évaluées par les professionnels pertinents ou les cliniques spécialisées (par ex., pédiatrie du développement, génétique clinique, psychiatrie, psychologie) afin d'identifier et de traiter leurs problèmes.

\section{Observations}

Le dépistage ne doit pas être considéré comme un diagnostic. Nous savons que dans certains endroits où il n'existe aucun service de diagnostic, les outils de dépistage ont parfois remplacé à tort le diagnostic approprié. Le dépistage a notamment pour objectif de repérer et de diriger vers un spécialiste les femmes enceintes susceptibles d'avoir un problème de consommation d'alcool et dont l'enfant risque d'être atteint de l'ETCAF. Plusieurs outils de dépistage de la consommation d'alcool se sont révélés efficaces en soins de santé primaires pour cerner l'abus d'alcool (p. ex., le TWEAK, le T-ACE, le CAGE, l'AUDIT, le S-MAST, le B-MAST) $)^{2,36-38}$.

Des preuves acceptables ${ }^{37,38}$ appuient l'utilisation des outils T-ACE et TWEAK pour identifier les femmes qui bénéficieraient d'une intervention en ce qui a trait à la consommation d'alcool pendant la grossesse. Si une femme ne peut s'abstenir d'alcool, elle devrait recevoir du soutien et être dirigée vers un centre de counselling et de traitement approprié. Cesser la consommation d'alcool à n'importe quel stade de la grossesse améliorera les chances du bébé. D'autres recherches sont en cours afin de mettre au point des instruments de dépistage appropriés adaptés en fonction

Tableau 2 : Critères du Code diagnostique à 4 chiffres pour l'ETCAF.

\begin{tabular}{|c|c|c|c|c|}
\hline Cote & Retard de croissance & $\begin{array}{l}\text { Traits faciaux } \\
\text { caractéristiques du SAF }\end{array}$ & $\begin{array}{l}\text { Lésion cérébrale ou } \\
\text { dysfonction }\end{array}$ & $\begin{array}{l}\text { Exposition à l'alcool } \\
\text { pendant la grossesse }\end{array}$ \\
\hline 4 & $\begin{array}{l}\text { Important } \\
\text { Taille et poids inférieurs au } \\
3^{\mathrm{e}} \text { percentile }\end{array}$ & $\begin{array}{l}\text { Très marqués } \\
\text { Tous les trois traits : } \\
\text { Ifp de } 2 \text { ou plus ET sous la } \\
\text { moyenne } \\
\text { Lèvre mince : cotée } 4 \text { ou } 5 \\
\text { Sillon naso-labial : } \\
\text { coté } 4 \text { ou } 5\end{array}$ & $\begin{array}{l}\text { Certaine } \\
\text { Preuve structurale ou } \\
\text { neurologique }\end{array}$ & $\begin{array}{l}\text { Risque élevé } \\
\text { Exposition à de hauts } \\
\text { niveaux confirmée }\end{array}$ \\
\hline 3 & $\begin{array}{l}\text { Modéré } \\
\text { Taille et poids inférieurs au } \\
10^{\mathrm{e}} \text { percentile }\end{array}$ & $\begin{array}{l}\text { Modéré } \\
\text { Généralement } 2 \text { des } 3 \text { traits }\end{array}$ & $\begin{array}{l}\text { Probable } \\
\text { Déficience importante } \\
\text { dans } 3 \text { domaines ou plus }\end{array}$ & $\begin{array}{l}\text { Un certain risque } \\
\text { Exposition confirmée } \\
\text { Niveau d'exposition } \\
\text { inconnu ou coté sous } 4\end{array}$ \\
\hline 2 & $\begin{array}{l}\text { Léger } \\
\text { Taille ou poids inférieurs au } \\
10^{\mathrm{e}} \text { percentile }\end{array}$ & $\begin{array}{l}\text { Léger } \\
\text { Généralement } 1 \text { des } 3 \text { traits }\end{array}$ & $\begin{array}{l}\text { Possible } \\
\text { Preuve de déficience, } \\
\text { mais cotée sous } 3\end{array}$ & $\begin{array}{l}\text { Inconnue } \\
\text { Présence ou absence } \\
\text { d'exposition non } \\
\text { confirmée }\end{array}$ \\
\hline 1 & $\begin{array}{l}\text { Aucun } \\
\text { Taille et poids supérieurs au } \\
10^{\mathrm{e}} \text { percentile }\end{array}$ & $\begin{array}{l}\text { Absents } \\
\text { Aucun des } 3 \text { traits }\end{array}$ & $\begin{array}{l}\text { Peu probable } \\
\text { Aucune preuve } \\
\text { structurale, neurologique } \\
\text { ou fonctionnelle de } \\
\text { déficience }\end{array}$ & $\begin{array}{l}\text { Aucun risque } \\
\text { Absence d'exposition de } \\
\text { la conception à la } \\
\text { naissance confirmée }\end{array}$ \\
\hline
\end{tabular}

Nota : fp = longueur de la fente palpébrale; $\mathrm{ET}$ = écart-type. 
du sexe et de la culture qui seraient utilisés pour toutes les femmes en âge de procréer ${ }^{38}$.

L'objet du dépistage chez les personnes à risque d'avoir subi les effets de l'exposition prénatale à l'alcool est de déterminer si des troubles d'apprentissage et de comportement seraient liés à l'exposition prénatale à l'alcool. Le dépistage pourrait être effectué par le biais du système d'éducation, du système de santé mentale, du système judiciaire ou des services sociaux. L'objet du dépistage devrait être de faciliter l'orientation vers une clinique de diagnostic et de souligner le besoin d'un soutien pour la mère.

Le réseau de diagnostic et de prévention du SAF (FAS Diagnostic and Prevention Network) a obtenu des résultats encourageants en appliquant l'outil de dépistage photographique des traits faciaux caractéristiques du SAF chez les enfants placés en foyer d'accueil et dans les populations d'enfants d'âge scolaire ${ }^{39}$. Toutefois, les dysmorphies faciales sont souvent absentes de la large gamme de l'ETCAF et, en bout de ligne, ont peu d'importance en comparaison avec les effets de l'exposition prénatale à l'alcool sur les fonctions du cerveau. Il importe cependant de noter que le phénotype facial est une anomalie médiane qui est l'indice le plus sensible et le plus spécifique de lésions cérébrales causées par l'alcool.

Toutes les personnes chez lesquelles on soupçonne des dysfonctions du cerveau devraient être dirigées vers un professionnel ou une clinique appropriée pour évaluation (par ex., la pédiatrie du développement, la génétique clinique, la psychiatrie, la psychologie). Étant donné la spécificité des cliniques pour l'ECTAF quant aux problèmes liés à l'exposition prénatale à l'alcool, les personnes n'ayant pas connu une telle exposition devraient être dirigées ailleurs, vers un professionnel pertinent ou une clinique appropriée pour l'évaluation, le traitement et le suivi.

\section{L'examen physique et le diagnostic différentiel}

L'évaluation des dysmorphies vise à repérer les personnes dont les traits sont caractéristiques d'une exposition prénatale à l'alcool et à repérer aussi les enfants dont les dysmorphies sont attribuables à d'autres causes. Parfois, les enfants atteints par les effets de l'exposition prénatale à l'alcool peuvent présenter des comorbidités résultant d'autres syndromes génétiques. En cas de doute, et là où il est possible de le faire, il est recommandé de procéder à une évaluation génétique et dysmorphologique.

Un examen physique général et un examen neurologique, y compris les mesures appropriées de la croissance et de la taille du crâne, l'évaluation des constatations caractéristiques et la documentation des anomalies (p. ex., la fissure palatine, la cardiopathie congénitale, les brides épicanthiques, le palais haut et arqué, les dents mal alignées ou anormales, l'hypertélorisme, la micrognathie, le dessin anormal de la chevelure, les plis de flexion palmaires anormaux, les lésions cutanées), sont requis afin de pouvoir exclure la présence d'autres troubles génétiques ou multifac- toriels qui pourraient avoir des caractéristiques rappelant celles du SAF ou du SAF partiel (Tableau 3).

Certains enfants auront des troubles neurologiques graves tels que la surdité, la cécité ou des convulsions qui devraient être évalués et documentés à titre d'éléments essentiels du profil de l'enfant. Ces caractéristiques ne permettent pas de faire la distinction entre les enfants qui ont été exposés à l'alcool et ceux qui ne l'ont pas été. Les traits faciaux caractéristiques du SAF sont attribuables à un effet particulier de la tératogenèse de l'éthanol qui modifie la croissance du milieu du visage et du cerveau. Les personnes exposées à d'autres agents embryotoxiques peuvent présenter un développement phénotypique semblable, mais non identique, au niveau du développement du visage, un retard de croissance, une fréquence plus élevée d'anomalies comportementales et du développement (pour plus de détails, voir Chudley et Longstaffe ${ }^{24}$ ). Toutefois, comme les traits faciaux caractéristiques du SAF ont été limités au raccourcissement des fentes palpébrales, à l'aplatissement du sillon naso-labial et à la minceur de la lèvre supérieure, il y a beaucoup moins de chevauchement avec les phénotypes faciaux associés à d'autres syndromes. La connaissance des antécédents d'exposition à l'alcool réduira la possibilité d'un diagnostic erroné de l'ETCAF.

Des enfants pourraient avoir besoin d'autres évaluations médicales pour cerner des troubles se présentant simultanément. Par exemple, les troubles du sommeil sont communs dans des cas d'exposition prénatale à l'alcool et des problèmes médicaux liés à l'apnée obstructive auraient pu échapper à une évaluation précédente. Des convulsions atypiques pourraient également se produire et des endocrinopathies pourraient exister en tant que facteur de comorbidité du retard de croissance. Ces personnes devraient être évaluées par les professionnels de la santé pertinents.

\section{2a. Croissance}

\section{Recommandation}

2.1 La croissance devrait être surveillée afin de dépister les déficiences. La présence d'un retard de croissance prénatal ou postnatal, défini comme une taille ou un poids égal ou inférieur au $10^{\mathrm{e}}$ percentile (écart-type de 1,5 au-dessous de la moyenne) ou un rapport poids-taille démesurément faible (égal ou inférieur au $10^{\mathrm{e}}$ percentile) en utilisant les normes appropriées. La constatation d'une croissance déficiente chez l'enfant exige la prise en compte de variables confusionnelles telles que la taille des parents et leur potentiel génétique et les conditions connexes (p. ex., le diabète gestationnel, l'état nutritionnel, la maladie).

\section{Observations}

Les enfants exposés à l'alcool avant la naissance peuvent avoir des retards de croissance prénatals ou postnatals. Ils peuvent être petits pour leur âge foetal in utero et la circonférence de leur tête, leur poids et leur taille peuvent rester 
au-dessous de la moyenne toute leur vie. De nombreux enfants peuvent avoir des paramètres normaux de croissance, mais risquent d'avoir des troubles cliniques d'apprentissage et de comportement et des déficits cognitifs importants à un stade ultérieur de leur développement. Si le fotus n'est pas exposé à l'alcool au troisième trimestre, les paramètres de croissance peuvent être normaux. Le diabète gestationnel peut aussi entraîner un accroissement du poids du foetus qui peut masquer le retard de croissance causé par l'exposition prénatale à l'alcool. De plus, si l'enfant est né dans une famille ou une communauté dans laquelle la taille «nor- male» est supérieure à celle de la population générale, le retard de croissance peut être masqué par une comparaison de l'enfant en fonction de paramètres de croissance normaux plutôt que des normes pour la communauté ${ }^{14}$. Les retards de croissance peuvent ne pas persister avec l'âge et il se peut que les dossiers sur la croissance infantile des adultes qui se présentent à une évaluation pour la première fois ne soient pas disponibles.

Des normes de croissance doivent être établies pour la population canadienne et pour les sous-populations qui pourraient différer de la population générale.

Tableau 3 : Syndromes ayant une constellation de caractéristiques communes au SAF.

\begin{tabular}{ll}
\hline Syndrome & Caractéristiques communes \\
\hline Syndrome d'Aarskog & $\begin{array}{l}\text { Yeux largement espacés, petit nez avec narines } \\
\text { antéversées, sillon naso-labial large, récession } \\
\text { médiofaciale. }\end{array}$
\end{tabular}

Caractéristiques de ce syndrome qui le différencient du SAF

Syndrome de BrachmandeLange ou de Cornelia deLange

Syndrome de Dubowitz

Syndrome foetal résultant d'un anticonvulsivant (incluant les syndromes fotaux attribuables à l'hydantoïne et au valproate) Effet de la phénylcétonurie maternelle sur le fœtus

Syndrome de Noonan

Embryopathie attribuable au toluène

Syndrome de Williams

Autres syndromes de délétion et de duplication chromosomiques
Sillon naso-labial long, vermillon de la lèvre supérieure mince, dos du nez déprimé, narines antéversées, microcéphalie.

Fentes palpébrales courtes, yeux largement espacés, brides épicanthiques, ptose variable (paupières retombantes) et blépharophimosis, microcéphalie.

Yeux largement espacés, dos du nez déprimé, récession médiofaciale, brides épicanthiques, vermillon de la lèvre supérieure mince.

Brides épicanthiques, fentes palpébrales courtes, sillon naso-labial long et mal formé, vermillon de la lèvre supérieure mince, microcéphalie.

Dos du nez bas, brides épicanthiques, yeux largement espacés, sillon naso-labial long.

Fentes palpébrales courtes, hypoplasie médiofaciale, sillon naso-labial aplati, vermillon de la lèvre supérieure mince, microcéphalie.

Fentes palpébrales courtes, narines antéversées, sillon naso-labial large et long, hypoplasie maxillaire, dos du nez déprimé, brides épicanthiques, microcéphalie.

Plusieurs individus ont les fentes palpébrales courtes, I'hypoplasie médiofaciale, le sillon nasolabial aplati.
Visage rond, fentes palpébrales bridées vers le bas, pousse des cheveux en $\mathrm{V}$ sur le front, oreilles en anse saillantes, difformité particulière des doigts en tension. Trait hérité lié au chromosome $x$. Défaut moléculaire identifié.

Sourcil unique en travers des yeux et du front (synophrys), cils longs, coins de la bouche tournés vers le bas, bras courts impliquant surtout le cubitus, taille très courte. Défaut moléculaire identifié.

Arcades sourcilières peu profondes, bout nasal large, clinodactylie.

Lèvre supérieure courbée, front large, petite bouche.

Glabelle saillante, petit nez relevé, visage rond.

Fentes palpébrales tournées vers le bas, bouche large avec sillon naso-labial bien formé, lèvre supérieure proéminente. Défaut moléculaire identifié.

Fontanelle antérieure large, dessin anormal de la chevelure, anomalies des oreilles.

Bouche large avec lèvres fortes et lèvre inférieure proéminente, structure étoilée de l'iris, rondeur périorbitaire, dysplasie des tissus conjonctifs, défaut cardiaque particulier de sténose aortique supravalvulaire chez plusieurs individus. Délétion chromosomique révélée lors de I'analyse du $7 q$ au moyen d'une sonde (test d'hybridation en fluorescence in situ).

Analyse chromosomique par analyse type et certains syndromes par analyse spécifique au moyen d'une sonde et du test $d$ 'hybridation en fluorescence in situ. 


\section{2b. Traits faciaux}

\section{Recommandations}

2.2 Les trois traits faciaux caractéristiques qui différencient les individus touchés par le SAF de ceux qui ne le sont pas sont les suivants :

- le raccourcissement des fentes palpébrales, égal ou inférieur au $3^{\mathrm{e}}$ percentile (écart-type de 2 au-dessous de la moyenne);

- l'aplatissement ou le lissage du sillon naso-labial, 4 ou 5 sur l'échelle de Likert à cinq points du Guide d'évaluation de la lèvre et du sillon naso-labial ${ }^{25,39}$;

- l'amincissement du vermillon de la lèvre supérieure, 4 ou 5 sur l'échelle de Likert à cinq points du Guide d'évaluation de la lèvre et du sillon naso-labial.

2.3 Les caractéristiques physiques connexes (anomalies telles que l'hypoplasie médiofaciale, la micrognathie, la position ou la formation anormale des oreilles, un palais haut et arqué, l'hypertélorisme, des brides épicanthiques, des anomalies des plis de flexion palmaires et de flexion des membres, et un nez court et tourné vers le haut) devraient être notées, mais elles ne contribuent pas à l'établissement du diagnostic.

2.4 Les traits faciaux devraient être mesurés pour tous les groupes d'âge. Si les traits faciaux d'un patient changent avec l'âge, le diagnostic devrait être fondé sur la période où les traits étaient les plus prononcés. Lors de l'établissement d'un diagnostic chez les adultes, il peut être utile de voir les photographies d'enfance.

\section{Observations}

Un profil crâno-facial caractéristique du SAF a d'abord été décrit par Jones et $\mathrm{Smith}^{40}$ en 1975 et plus tard raffiné par Astley, Clarren et d'autres ${ }^{25,32,39}$. Les personnes atteintes du SAF ont des fentes palpébrales raccourcies, une lèvre supérieure mince et un sillon naso-labial mal dessiné (Figure 1). La longueur de la fente palpébrale, le sillon naso-labial et la lèvre supérieure varient selon la race et l'âge. Il faut disposer de données anthropométriques sur la croissance et le visage pour les populations spécifiques, puisque la précision et la spécificité de l'évaluation seront réduites sans l'usage de normes appropriées. Certains traits caractéristiques du SAF (p. ex., le vermillon de la lèvre ou le sillon naso-labial) peuvent devenir moins reconnaissables avec l'âge, ce qui rend plus difficile l'établissement d'un diagnostic précis chez les groupes plus âgés, mais les traits faciaux devraient toujours être mesurés. Il faudra mener d'autres recherches longitudinales afin d'établir la corrélation entre les changements dans ces caractéristiques physiques chez les adolescents et les adultes pour lesquels un diagnostic de SAF ou de SAF partiel a été posé.

Sans formation, la longueur des fentes palpébrales (Figure 2) est difficile à mesurer avec précision. Thomas et ses collègues $^{41}$ ont publié des normes de longueur des fentes palpébrales de l'âge foetal de 29 semaines jusqu'à l'âge de 14 ans. Les opinions varient quant à l'opportunité des normes ${ }^{41-44}$, mais on convient généralement que toutes comportent une certaine part de lacunes.

Deux graphiques sur la longueur de la fente palpébrale sont présentés à l'Annexe 2. Il existe des différences entre elles. Les deux analyses ont utilisé des sujets blancs nord-américains; des normes pour les autres populations au Canada ne sont pas actuellement disponibles. L'Annexe 2-1 pourrait être plus fiable pour mesurer la longueur de la fente palpébrale à l'aide d'une règle en plastique (selon l'expérience de l'un des auteurs). L'Annexe 2-2 pourrait être plus fiable si l'on utilise un compas à calibrer (selon l'expérience de l'un des auteurs). Les rangs centiles des deux figures concordent jusqu'à l'âge de 7 ans, après quoi l'Annexe 2-2 indique des fentes palpébrales plus longues chez les enfants plus âgés et les adolescents que l'Annexe 2-1. Nous croyons que ces différences s'expliquent par les techniques de mesure employées. Puisque le compas à calibrer n'est pas un outil retrouvé ordinairement dans la plu-

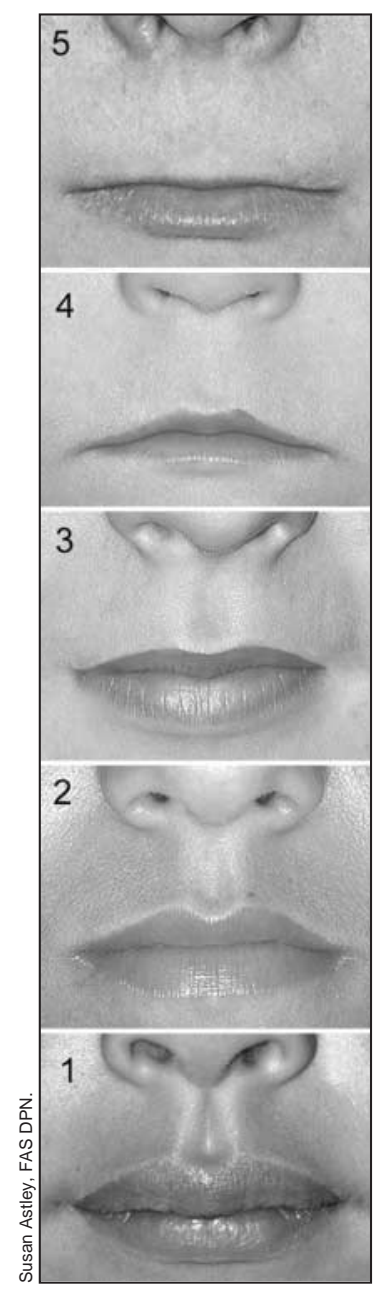

Figure 1 : Guide d'évaluation de la lèvre et du sillon naso-labial. Ce guide sert d'échelle visuelle à cing points pour mesurer I'aplatissement du sillon naso-labial et la minceur de la lèvre supérieure. Chaque trait est mesuré indépendamment de l'autre. Par exemple, un individu peut avoir un sillon naso-labial coté 5 et une lèvre supérieure cotée 1. 
part des cliniques médicales, nous recommandons l'usage d'une règle de plastique flexible clair.

Il faut établir des normes à jour pour tous les âges et les sous-populations. Astley et Clarren ${ }^{25,39}$ ont élaboré des normes pour l'évaluation de la lèvre et du sillon naso-labial en utilisant leur guide visuel. Des guides d'évaluation de la lèvre et du sillon naso-labial ont été élaborés pour les populations caucasienne et noire américaines, mais aucune norme n'est actuellement disponible pour les autres populations (Figure 2).

\section{L'évaluation du comportement neurologique}

\section{Recommandations}

3.1 Les domaines suivants devraient être évalués :

a. Troubles neurologiques graves et légers (y compris au niveau de la fonction sensori-motrice).

b. Structure du cerveau (circonférence occipitofrontale, imagerie par résonance magnétique, etc.).

c. Cognition (QI).

d. Communication : réceptive et expressive.

e. Résultats scolaires.

f. Mémoire.

g. Fonctionnement exécutif et raisonnement abstrait.

h. Déficience de l'attention et hyperactivité.

i. Comportement adaptatif, aptitudes sociales, communication sociale.

3.2 L'évaluation devrait inclure et comparer des tâches élémentaires et des tâches complexes dans chaque domaine, selon les besoins.

3.3 Les domaines doivent être évalués comme s'ils constituaient des entités indépendantes, mais lorsqu'il y a chevauchement, il faut exercer son jugement clinique pour pouvoir décider combien de domaines sont touchés.

3.4 Un domaine est considéré comme «atteint d'anomalie» lorsque, selon une mesure normalisée :

a. les mesures sont d'un écart-type de 2 ou plus au-

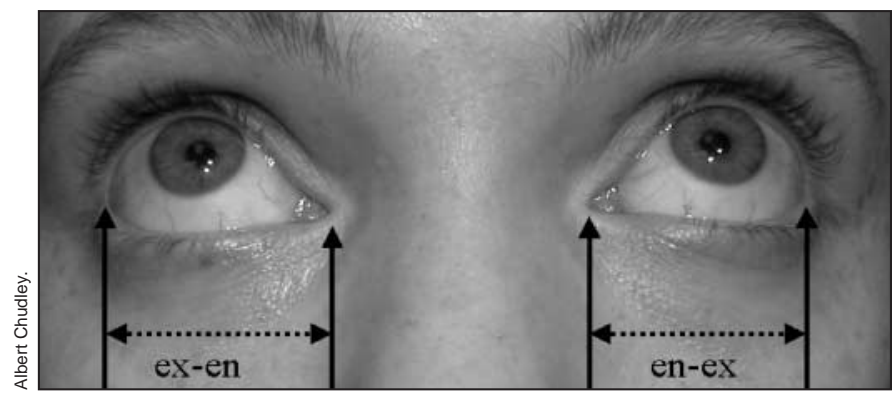

Figure 2 : Longueur de la fente palpébrale. Repères utilisés pour mesurer la fente palpébrale des deux yeux. Identifiez le coin intérieur ou encanthion (en) et le coin extérieur ou excanthion (ex) de chaque œil. Demandez à l'individu de regarder vers le haut afin que les ex apparaissent clairement. À l'aide d'une règle souple et claire, mesurez la longueur de chaque intervalle ex-en sur le plan horizontal immédiatement au-dessous de l'œil, en prenant soin de ne pas toucher l'œil ou les cils. Reportez les résultats sur un nomogramme afin de déterminer le centile ou l'écart-type pour chaque œil. dessous de la moyenne, ou

b. il y a une différence d'écart-type d'au moins 1 entre les sous-domaines. Par exemple :

i. aptitudes verbales c. aptitudes non verbales aux tests de QI normalisés;

ii. langage expressif c. langage réceptif;

iii. mémoire verbale c. mémoire visuelle; ou

c. il y a une différence d'écart-type d'au moins 1,5 à 2 entre des sous-tests d'une mesure, en fonction du degré de fiabilité de la mesure en question et de sa variabilité normale dans la population.

3.5 Dans les domaines pour lesquels des mesures normalisées ne sont pas disponibles, on pose le jugement clinique de «dysfonction importante», en tenant compte du fait que des variables importantes, y compris l'âge de l'enfant, les facteurs de santé mentale, les facteurs socioéconomiques et les familles ou milieux de vie perturbés (p. ex., les placements multiples dans des familles d'accueil, les antécédents de mauvais traitements et de négligence) peuvent toucher le développement, mais n'indiquent pas la présence de lésion cérébrale.

3.6 La preuve d'une déficience dans trois domaines est nécessaire pour un diagnostic, mais une évaluation complète devrait inclure les forces et les faiblesses dans chaque domaine.

3.7 Dans le cas de certains enfants à risque (p. ex., d'âge préscolaire) qui ont été exposés à l'alcool, mais chez lesquels aucune déficience mesurable n'apparaîtra dans les domaines du cerveau ou qui pourraient être trop jeunes pour subir des tests dans tous les domaines, le diagnostic devrait être différé. Toutefois, il convient d'effectuer une évaluation du développement afin de cerner les domaines dans lesquels une intervention précoce s'impose.

Des exemples des tests les plus couramment utilisés pour évaluer les domaines et de leurs critères figurent à l'Annexe 3 .

\section{Observations}

Les comptes rendus de recherche ont documenté un éventail de résultats sur les plans cognitif et comportemental associés à l'exposition prénatale à l'alcool. Les études contemporaines ont signalé certains de ces résultats en l'absence des caractéristiques physiques du SAF. Pour le moment, on n'a pas trouvé de profil modal des aptitudes qui s'applique exclusivement à l'exposition à l'alcool, soit observé chez toutes les personnes exposées à l'alcool avant la naissance, ou puisse se distinguer de celui que l'on retrouve chez les personnes atteintes d'autres troubles neurologiques du comportement. De plus, toutes les déficiences qui peuvent être retrouvées chez un enfant qui a été exposé à l'alcool avant la naissance ne sont pas nécessairement causées uniquement par cette exposition. Une analyse par des experts des troubles neurologiques du développement causés par toute une gamme de tératogènes et d'affections congénitales n'est pas par-venue à faire le consensus quant aux déficiences de base associées uniquement à l'ETCAF'.

La recherche et l'expérience ont démontré que les carac- 
téristiques de l'ETCAF sont complexes et comportent de nombreuses facettes, trouvant leur origine dans des atteintes cérébrales organiques causées par l'alcool, mais ayant des interactions avec d'autres influences sur le plan génétique ou autre. Au cours de la vie de la personne atteinte, ces caractéristiques peuvent être exacerbées ou atténuées par ses expériences dans son milieu.

Pour établir le diagnostic du SAF, des caractéristiques telles que la microcéphalie, les anomalies structurales (décelées par scintigraphie cérébrale) et les troubles neurologiques graves sont considérés comme des preuves d'atteintes cérébrales organiques. Nous croyons qu'un QI normal-faible ou limite et des troubles neurologiques légers ne sont pas des preuves suffisantes de lésion cérébrale étant donné qu'ils sont fréquents dans la population générale. Des caractéristiques telles que les difficultés d'apprentissage, les troubles déficitaires de l'attention avec hyperactivité et les déficiences dans les facultés d'adaptation, la mémoire, le langage de plus haut niveau et le raisonnement abstrait sont souvent constatées chez les enfants qui ont été exposés à l'alcool avant la naissance, mais aussi chez ceux dont le cas relève d'une autre étiologie. L'étiologie de ces déficiences peut être multifactorielle et elles peuvent aussi être attribuées à l'hérédité ou à des expériences postnatales.

Le Code diagnostic à 4 chiffres d'évaluation de l'ETCAF est fondé sur des niveaux de certitude, selon le jugement du clinicien, que les problèmes cognitifs et comportementaux de la personne sont attribuables à une lésion cérébrale. Une cote plus élevée peut indiquer une manifestation plus grave de déficience fonctionnelle, une performance irrégulière d'un domaine à l'autre, ou une certitude fondée sur des déficiences dans plusieurs domaines ${ }^{25,39}$. La détermination est fondée sur la preuve objective de «déficiences ou d'écarts importants dans plusieurs domaines du fonctionnement du cerveau».

$\mathrm{L}^{\prime} \mathrm{IOM}^{4}$ exige également la «preuve d'un ensemble complexe d'anomalies comportementales ou cognitives qui ne peuvent être expliquées par les antécédents familiaux ou le milieu seulement, telles que des difficultés d'apprentissage, un rendement scolaire déficient, un mauvais contrôle des impulsions, des problèmes de perception sociale, des déficiences dans le langage réceptif et expressif de haut niveau, une faible capacité d'abstraction ou de métacognition, des déficiences particulières en mathématiques, ou des problèmes de mémoire, d'attention ou de jugement», mais il est beaucoup moins précis que le Code diagnostique à 4 chiffres en ce qui concerne les critères utilisés pour déterminer la présence des déficiences.

Nous avons adapté la méthodologie du Code diagnostique à 4 chiffres en ce qui concerne la détermination des domaines, la gravité des déficiences et la certitude de lésion cérébrale. La recherche actuelle démontre que, sur le plan neurologique de comportement, il y a chevauchement entre les résultats pour les groupes de diagnostic du SAF et des TNDLA lorsque l'on compare les données neuropsychologiques $^{45}$. De plus, nous croyons qu'une seule caractéristique telle que la microcéphalie n'est pas un indicateur suffisant de lésion cérébrale aux fins du diagnostic du SAF étant donné qu'elle peut être attribuable à des différences géné- tiques ou ethniques qui ne sont pas prises en considération dans les normes physiques actuellement disponibles. Nous sommes préoccupés par le fait qu'il risque d'y avoir surdiagnostic de SAF si la preuve de lésion cérébrale est fondée sur un seul indicateur, comme le permettent tant le Code diagnostique à 4 chiffres que les modèles de l'IOM. Une personne atteinte de troubles neurologiques graves ou d'anomalies structurales du cerveau (c.-à-d., une véritable lésion cérébrale) aura sans doute aussi d'autres déficiences fonctionnelles dans les domaines énumérés. Un diagnostic de SAF complet sera toujours possible même si l'on combine les critères du SAF complet et des TNDLA dans ce système harmonisé.

Bien que les domaines soient considérés comme des entités distinctes et indépendantes, il y a évidemment un chevauchement. Par exemple, un écart entre les aptitudes verbales et les aptitudes non verbales dans un test de QI peut indiquer une déficience particulière du langage. S'il y a déficience du langage, des déficiences de la mémoire verbale peuvent-elles être considérées comme un domaine additionnel? Une déficience du langage indique-t-elle une lésion cérébrale si l'enfant a vécu une période prolongée de privation sociale? Afin d'accroître la certitude que les déficiences dans le domaine sont attribuables à une lésion cérébrale, nous recommandons d'établir le seuil de l'écart-type à 2 au-dessous de la moyenne dans les tests normalisés et d'attribuer une cote de 3 (dysfonction importante) dans le Code diagnostique à 4 chiffres. L'obtention de ces résultats dans trois domaines donnerait la cote de 3 pour le cerveau : «dysfonction probable du cerveau».

Nous sommes conscients du fait que, dans les pratiques de neuropsychologie, un écart-type de 1,5 au-dessous de la moyenne peut indiquer de légères déficiences. En utilisant le Code diagnostique à 4 chiffres, les domaines seraient cotés comme «moyennement dysfonctionnels», ce qui pourrait donner une cote de 2 pour le cerveau : «dysfonction possible du cerveau». Ces conclusions plus subtiles constituent une partie importante du profil de l'individu. Aux fins du diagnostic, toutefois, et afin de s'assurer que les cotes représentent des dommages causés par l'alcool, nous recommandons le seuil le plus radical. L'équipe multidisciplinaire qui examine les données et pose un jugement clinique est essentielle pour l'établissement d'un diagnostic précis étant donné que les aspects qualitatifs du rendement sont également importants. Le profil du diagnostic est dynamique et peut changer au cours du temps, et il se peut donc que les individus atteints ou que l'on croit être atteints aient à subir plusieurs évaluations. Les services ne doivent pas être fondés sur le diagnostic même, mais plutôt sur le profil des fonctions et dysfonctions cérébrales.

\section{Traitement et suivi}

\section{Recommandations}

4.1 Il est crucial de renseigner le patient et les membres de sa famille sur les aspects de l'ETCAF, ainsi que de discuter des tensions psychosociales potentielles au sein de la famille à la 
suite du diagnostic. La communication doit être sensible au milieu culturel et se faire dans un langage approprié.

4.2 Un membre de l'équipe de diagnostic doit faire le suivi des évaluations diagnostiques et des plans de traitement dans un délai raisonnable afin de s'assurer que toutes les recommandations ont été abordées.

4.3 Les personnes ayant reçu un diagnostic et leurs familles devraient être mises en communication avec les ressources et les services qui amélioreront leur sort. Toutefois, quand les services offerts dans la collectivité sont limités, une personne ne devrait pas être privée d'une évaluation à des fins de diagnostic et de traitement. Souvent un diagnostic posé chez un individu devient l'incitatif menant au développement des ressources.

\section{Les antécédents de consommation d'alcool de la mère pendant la grossesse}

\section{Recommandations}

5.1 L'exposition prénatale confirmée à l'alcool exige la confirmation de la consommation d'alcool par la mère pendant la grossesse index, fondée sur une observation clinique fiable, une autodéclaration, les rapports d'une source fiable ou des dossiers médicaux documentant un taux d'alcool dans le sang, un traitement de l'alcoolisme ou d'autres problèmes d'ordre social, juridique ou médical liés à la consommation d'alcool pendant la grossesse.

5.2 Le nombre et le type de boissons alcoolisées consommées (dose), les habitudes de consommation et la fréquence de la consommation devraient tous être documentés.

5.3 Pris isolément, les ouï-dire, le style de vie, la consommation d'autres drogues, ou les antécédents de consommation d'alcool lors de grossesses précédentes ne peuvent révéler les habitudes de consommation d'alcool pendant la grossesse index. Toutefois, la présence simultanée de plusieurs troubles, d'agents stresseurs psychosociaux importants et d'exposition prénatale à d'autres substances (p. ex., la cigarette, les drogues licites ou illicites) lors de la grossesse index et de grossesses précédentes devrait tout de même être consignée, étant donné que l'on sait que les interactions entre ces variables influent sur la gravité des affections au terme de la grossesse tant pour la mère que pour le nouveau-né.

\section{Observations}

Il est essentiel de recueillir des renseignements fiables sur la consommation d'alcool par la mère afin de pouvoir établir un diagnostic précis. Lors de l'entrevue, il est important de s'informer sur la consommation d'alcool de la mère pendant la période où elle ne savait pas encore qu'elle était enceinte. Certaines femmes ne considèrent pas comme importante leur consommation d'alcool antérieure et plusieurs d'entre elles la sous-déclarent. Il s'impose de recevoir la formation voulue pour apprendre comment obtenir ces renseignements sans juger la personne ou l'intimider.
Les données d'enquêtes canadiennes suggèrent que le nombre de femmes qui déclarent avoir consommé de l'alcool pendant leur grossesse a diminué. Les données de l'Enquête nationale sur la santé de la population de 1994-1995 ${ }^{46}$ et de l'Enquête longitudinale nationale sur les enfants et les jeunes de $1994-1995^{47}$ indiquent aussi que $17 \%$ à $25 \%$ des femmes ont bu de l'alcool à un certain stade de leur grossesse, et que $7 \%$ à $9 \%$ des femmes en ont consommé tout au long de leur grossesse. Selon les résultats de l'Enquête longitudinale nationale sur les enfants et les jeunes de 1998-19998, 14,4 \% des femmes ont consommé de l'alcool à un certain stade de leur grossesse et $4,9 \%$ des femmes en ont consommé tout au long de leur grossesse (3\% ont déclaré avoir eu des épisodes de consommation excessive d'alcool pendant leur grossesse). Dans l'Enquête sur les citoyens des Premières nations qui vivent dans les réserves, réalisée à l'automne $2002^{49}, 53$ \% des répondantes ont affirmé qu'il est important pour les femmes de réduire ou de cesser leur consommation d'alcool pour avoir un bébé en santé.

L'évaluation de ce qui constitue une «exposition importante à l'alcool» est souvent difficile. L'IOM décrit l'exposition importante à l'alcool comme «des habitudes de consommation excessive caractérisées par une consommation régulière substantielle ou par des épisodes de consommation excessive» (le National Institute on Alcohol, Alcoholism, and Alcohol Abuse définit la consommation excessive comme étant 5 verres ou plus par épisode, 5 jours ou plus au cours des derniers 30 jours $^{36}$ ). Ces habitudes peuvent se manifester par de fréquents épisodes d'intoxication, le développement d'une tolérance à l'alcool ou de symptômes de sevrage, des problèmes sociaux liés à la consommation d'alcool, des problèmes judiciaires liés à la consommation d'alcool, un comportement physiquement dangereux lors des épisodes de consommation d'alcool, ou des problèmes médicaux liés à l'alcool tels que les maladies du foie. À mesure que d'autres recherches seront effectuées et que, le cas échéant, de moindres quantités d'alcool ou d'autres habitudes de consommation seront associées à des ACLA ou à des TNDLA, ces habitudes de consommation d'alcool devraient être incorporées dans les critères de diagnostic ${ }^{4}$.

\section{Critères de diagnostic du SAF, du SAF partiel et des TNDLA}

\section{Recommandations}

6.1 Les critères de diagnostic du syndrome d'alcoolisation fœtale, après avoir exclu d'autres diagnostics, sont les suivants :

A. Preuve d'un retard de croissance prénatal ou postnatal, dans au moins un des aspects suivants :

a. poids ou longueur à la naissance égal ou inférieur au $10^{\mathrm{e}}$ percentile pour l'âge fotal;

b. taille ou poids égal ou inférieur au $10^{\mathrm{e}}$ percentile pour l'âge;

c. rapport poids-taille trop faible (égal ou inférieur au $10^{\mathrm{e}}$ percentile). 
B. Présence simultanée, à n'importe quel âge, de toutes trois des anomalies faciales suivantes:

a. fentes palpébrales courtes (d'un écart-type de 2 ou plus au-dessous de la moyenne);

b. sillon naso-labial lisse et aplati (coté 4 ou 5 selon le Guide d'évaluation de la lèvre et du sillon nasolabial);

c. lèvre supérieure mince (cotée 4 ou 5 selon le Guide d'évaluation de la lèvre et du sillon nasolabial).

C. Preuve de déficiences dans trois des domaines du système nerveux central ou plus, notamment : les troubles neurologiques légers et profonds; la structure du cerveau; la cognition; la communication; le rendement scolaire; la mémoire; le fonctionnement exécutif et le raisonnement abstrait; la déficience de l'attention et hyperactivité; le comportement adaptatif, les compétences sociales, la communication sociale.

D. Exposition de la mère à l'alcool confirmée (ou non confirmée).

6.2 Les critères de diagnostic du syndrome d'alcoolisation fœetale partiel, après avoir exclu d'autres diagnostics, sont les suivants :

A. Présence simultanée, à n'importe quel âge, de deux des anomalies faciales suivantes:

a. fentes palpébrales courtes (d'un écart-type de 2 ou plus au-dessous de la moyenne);

b. sillon naso-labial lisse ou aplati (coté 4 ou 5 selon le Guide d'évaluation de la lèvre et du sillon nasolabial);

c. lèvre supérieure mince (cotée 4 ou 5 selon le Guide d'évaluation de la lèvre et du sillon naso-labial).

B. Preuve de déficiences dans trois des domaines du système nerveux central ou plus, notamment : les troubles neurologiques légers et profonds; la structure du cerveau; la cognition, la communication; le rendement scolaire; la mémoire; le fonctionnement exécutif et le raisonnement abstrait; la déficience de l'attention et hyperactivité; le comportement adaptatif, les compétences sociales, la communication sociale.

C. Exposition de la mère à l'alcool confirmée.

6.3 Les critères de diagnostic des troubles neurologiques du développement liés à l'alcool, après avoir exclu d'autres diagnostics, sont les suivants :

A. Preuve de déficiences dans trois ou plus des domaines du système nerveux central : les troubles neurologiques légers et profonds; la structure du cerveau; la cognition; la communication; le rendement scolaire; la mémoire; le fonctionnement exécutif et le raisonnement abstrait; la déficience de l'attention et hyperactivité; le comportement adaptatif, les compétences sociales, la communication sociale.

B. Exposition de la mère à l'alcool confirmée.

6.4 L'expression anomalies congénitales liées à l'alcool (ACLA) ne devrait être utilisée ni comme une expression générique ni comme un terme de diagnostic, pour désigner l'ensemble des effets causés par l'alcool. Les ACLA constituent une série d'anomalies congénitales, incluant des malformations et des dysplasies, et il faut s'assurer d'utiliser l'expression à juste titre (Tableau 1).

\section{Observations}

Notre définition du SAF partiel diffère des critères publiés par l'IOM ${ }^{4}$. Lorsqu'on sait qu'il y a eu une exposition prénatale importante à l'alcool et que l'on constate un retard de croissance important et des traits faciaux caractéristiques mais sans preuve d'atteinte cérébrale, un diagnostic de SAF partiel pourrait être posé en utilisant les critères de l'IOM. Selon nous, l'utilisation de l'expression SAF partiel en l'absence de déficiences mesurables du cerveau pourrait causer un préjudice à l'individu, étant donné qu'un tel diagnostic de SAF partiel suggère des dysfonctions du cerveau. Si l'on constate certains traits faciaux caractéristiques et un retard de croissance chez un enfant âgé de moins de six ans, sans qu'il ait de problèmes de développement ou de comportement importants, il serait prudent de dire que l'enfant risque éventuellement d'avoir des problèmes d'apprentissage et de comportement à cause de son exposition à l'alcool avant la naissance. Aucun diagnostic lié à l'alcool ne devrait être posé, mais l'enfant devrait être suivi par le médecin de famille ou un fournisseur de soins de santé et les déficiences devraient être documentées au moyen d'une évaluation neurologique du développement.

Dans l'expression SAF partiel, le terme «partiel» ne signifie pas que les déficiences des individus atteints les touchent moins dans leur fonctionnement quotidien que les individus pour lesquels un diagnostic de SAF est posé, étant donné que les déficiences dans le fonctionnement du cerveau peuvent être semblables.

\section{Harmonisation des approches de l'Institute of Medicine (IOM) et du Code diagnostique à 4 chiffres}

\section{Recommandations}

7.1 L'approche du Code diagnostique à 4 chiffres devrait être utilisée pour décrire, évaluer et mesurer de façon objective l'exposition à l'alcool et les dommages causés à la croissance, aux traits du visage et au cerveau. Il faudrait noter le Code à 4 chiffres de chaque évaluation, qui pourra être utile lors du suivi et aux fins de recherches.

7.2 La terminologie des critères de l'IOM devrait être utilisée pour décrire le diagnostic.

\section{Observations}

La façon dont nous recommandons d'harmoniser les critères de l'IOM et le Code diagnostique à 4 chiffres est il- 
lustrée aux Tableau 4 et Tableau 5. La catégorie des ACLA est d'une utilité limitée dans le diagnostic, mais nous reconnaissons que l'alcool est tératogène et peut causer des anomalies congénitales lorsque le fœtus y est exposé pendant les périodes critiques de développement. Toutefois, en l'absence d'autres caractéristiques du SAF ou de déficiences du cerveau, il est difficile d'attribuer une cause.

\section{Recherches futures relatives aux lignes directrices concernant le diagnostic}

L'absence ou le manque de disponibilité de renseignements ou de preuves dans les domaines clés limite l'efficacité du processus de diagnostic en général. Les domaines clés comprennent l'élaboration de normes canadiennes sur la croissance et les données anthropométriques pour tous les âges et les groupes ethno-culturels. Également nécessaires sont le développement et la validation d'outils de dépistage qui sont spécifiques et sensibles à l'exposition prénatale à l'alcool. Ces outils de dépistage devraient pouvoir être adaptés à divers contextes, devraient être appropriés au milieu culturel et devraient mener à des orientations correctes pour le diagnostic et l'évaluation.

\section{Nouveaux enjeux}

\section{Biomarqueurs}

Souvent, les femmes ne se souviendront pas de la quantité d'alcool qu'elles ont consommée pendant leur grossesse ni de la fréquence de leur consommation. Certaines femmes peuvent également sous-estimer leur consommation ou refuser d'admettre qu'elles ont bu de l'alcool pendant leur grossesse. L'on sait que les dossiers médicaux sont incomplets en ce qui concerne les antécédents de consommation d'alcool de la mère. Il n'existe à l'heure actuelle aucun moyen fiable de confirmer la con- sommation d'alcool par la mère en utilisant des marqueurs biochimiques pendant la grossesse. Des concentrations élevées d'acétaldéhyde dans le sang total, une transferrine déficiente en glucides, le gamma-glutamyl transpeptidase et le volume de globules rouges peuvent être des marqueurs utiles chez les femmes enceintes ${ }^{50}$.

L'on mène actuellement des études afin de déterminer l'utilité des mesures de la concentration de l'ester éthylique d'acide gras dans le méconium à titre de marqueur pour l'exposition prénatale à l'alcool ${ }^{51-53}$. Ce marqueur ne sera utile que si l'on peut établir que les concentrations d'ester éthylique d'acide gras dans le méconium permettent de prédire les résultats au niveau du développement. Les tests sur le méconium pourraient attirer l'attention des fournisseurs de soins sur les enfants qui risquent d'avoir été exposés aux effets de l'alcool et permettre ainsi un contrôle, une intervention et une prévention appropriés. L'utilisation de marqueurs biologiques chez le bébé pouvant révéler une consommation d'alcool par la mère soulève des questions d'éthique quant au consentement éclairé.

Des innovations récentes ont mené au développement de la scintigraphie de surface au laser, une méthode non invasive d'obtenir des images en trois dimensions ${ }^{33,34}$. Cette technique est prometteuse pour l'analyse des traits faciaux associés à l'exposition prénatale à l'alcool, mais elle demeure encore un outil de recherche ${ }^{33,34}$.

\section{Régions éloignées et rurales}

La disponibilité de services de diagnostic est limitée dans les régions rurales et éloignées. La communauté peut ne pas avoir accès à une équipe de diagnostic ou à des ressources et des services. En attendant que des équipes de diagnostic régionales soient formées, il pourrait être utile de recourir à la télémédecine pour les diagnostics, les consultations et la formation à distance ${ }^{30}$. Les récents progrès de l'imagerie numérique et des analyses assistées par ordi-

\section{Tableau 4 : Harmonisation de la nomenclature de I'Institute of Medicine (IOM) et des cotes du Code diagnostique à 4 chiffres pour la croissance, le visage, le cerveau et les antécédents de consommation d'alcool.}

\begin{tabular}{|c|c|c|c|c|}
\hline \multirow[b]{2}{*}{ Nomenclature de I'IOM } & \multicolumn{4}{|c|}{ Cotes du Code diagnostique à 4 chiffres } \\
\hline & Retard de croissance & $\begin{array}{l}\text { Phénotype facial } \\
\text { du SAF }\end{array}$ & $\begin{array}{l}\text { Dommage au SNC } \\
\text { ou dysfonction }\end{array}$ & $\begin{array}{l}\text { Antécédents de } \\
\text { consommation d'alcool } \\
\text { pendant la grossesse }\end{array}$ \\
\hline SAF (avec exposition confirmée) & 2,3 ou 4 & 3 ou 4 & 3 ou 4 & 3 ou 4 \\
\hline $\begin{array}{l}\text { SAF } \\
\text { (sans exposition confirmée) }\end{array}$ & 2,3 ou 4 & 3 ou 4 & 3 ou 4 & 2 \\
\hline $\begin{array}{l}\text { SAF partiel (avec exposition } \\
\text { confirmée)* }\end{array}$ & $1,2,3$ ou 4 & 2,3 ou 4 & 3 ou 4 & 3 ou 4 \\
\hline ACLA (avec exposition confirmée) & $1,2,3$ ou 4 & 1 ou 2 & $\begin{array}{l}3 \text { ou } 4 \\
(2 \text { pour }<6 \text { ans })\end{array}$ & 3 ou 4 \\
\hline
\end{tabular}

Nota : $\mathrm{ACLA}=$ anomalies congénitales liées à l'alcool; SNC = système nerveux central; SAF = syndrome d'alcoolisation fœetale.

Source : Mis au point par Kwadwo Asante et Julianne Conry.

*Tout code final à 4 chiffres pouvant être établi à partir de ces combinaisons de chiffres et qui n'est pas aussi un code du SAF correspond au SAF partiel. Les combinaisons de caractéristiques faciales cotées 2 et réunissant deux phénotypes faciaux significatifs répondent aussi aux critères du SAF partiel. 
nateur pour le dia-gnostic des traits caractéristiques du SAF semblent prometteuses pour l'évaluation des traits faciaux associés à l'exposition prénatale à l'alcool ${ }^{32,33,4}$.

\section{Diagnostic chez les adultes}

L'établissement d'un diagnostic chez les adultes représente un défi dans tous les aspects du diagnostic. Les traits physiques peuvent changer avec le temps, il peut y avoir un rattrapage dans la croissance et l'effet cumulé des influences environnementales peut altérer l'évaluation des fonctions du cerveau. Les antécédents de l'adulte peuvent inclure des traumatismes crâniens, l'abus d'alcool et d'autres drogues, et des troubles mentaux. Bien que des tests soient disponibles pour les divers domaines, les cliniciens œuvrant avec la population des adultes atteints par l'ETCAF constatent que souvent les tests ne sont pas sensibles aux conditions réelles. En plus des données requises pour établir le diagnostic, l'évaluation doit comporter des composantes additionnelles telles que l'alphabétisation, les capacités au calcul, l'employabilité et la qualité de vie qui relèvent du domaine des capacités d'adaptation. Le clinicien ne devrait pas se fier uniquement à l'autodéclaration de l'individu touché par l'alcool et devrait vérifier ses antécédents et ses capacités auprès d'une source fiable.

\section{Conclusion}

L'évaluation diagnostique de l'exposition prénatale à l'alcool vise l'individu atteint, sa mère naturelle et ses frères et sœurs qui pourraient aussi être atteints. Plutôt que d'étiqueter le patient, le diagnostic offre un plan directeur pour une intervention précoce. Le plan de traitement et sa mise en œuvre, visant les besoins particuliers de l'individu et de sa famille, sont des éléments importants du diagnostic.

Les présentes lignes directrices et recommandations ont été élaborées en parallèle et en consultation avec un comité américain chargé de la même tâche ${ }^{54}$. Les défis que posent la prévention, le diagnostic et l'intervention chez les individus atteints par l'ETCAF évoluent sans cesse. L'on mène actuellement des recherches pour déterminer si des outils tels que les nouvelles techniques d'imagerie du cerveau, les biomarqueurs et les techniques de microréseaux d'ADN peuvent aider à améliorer la précision et la fiabilité des diagnostics liés à l'alcool et le traitement.

Nous espérons que les présentes lignes directrices et recommandations aideront à faciliter la formation des professionnels de la santé, à améliorer l'accès aux services de diagnostic et à faciliter l'orientation vers une intervention ou un traitement pour toutes les personnes qui vivent avec cette incapacité et pour leur famille.
Tableau 5 : Comparaison des méthodes de I'Institute of Medicine (IOM) et du code diagnostique à 4 chiffres pour un diagnostic de SAF.

\begin{tabular}{|c|c|c|}
\hline Caractéristique & $\mathrm{IOM}$ & Code diagnostique à 4 chiffres \\
\hline \multicolumn{3}{|l|}{ Visage } \\
\hline Nombre de caractéristiques requises & Non précisé & 3 sur 3 \\
\hline Lèvre supérieure mince (aplatie) & Oui & Oui \\
\hline Sillon naso-labial aplati & Oui & Oui \\
\hline Aplatissement médiofacial & Oui & Non \\
\hline Fentes palpébrales raccourcies & Oui & Oui \\
\hline Autres caractéristiques & $?$ & Non \\
\hline \multicolumn{3}{|l|}{ Croissance } \\
\hline Nombre de caractéristiques requises & 1 & 1 \\
\hline Faible poids à la naissance seulement & $\begin{array}{l}\text { Oui, percentile non } \\
\text { précisé }\end{array}$ & Non \\
\hline Décroissance du poids avec le temps & Oui & Non \\
\hline Poids faible par rapport à la taille & Oui & Non \\
\hline Petite taille et faible poids & Non & Oui, $\leq$ au $10^{\mathrm{e}}$ percentile \\
\hline \multicolumn{3}{|l|}{ Dysfonction du système nerveux central } \\
\hline Nombre de caractéristiques requises & $\begin{array}{l}1 \text { caractéristique } \\
\text { structurale ou } \\
\text { neurologique }\end{array}$ & $\begin{array}{l}1 \text { caractéristique structurale ou } \\
\text { neurologique OU } \\
\text { déficience importante de la } \\
\text { fonction dans } 3 \text { domaines }\end{array}$ \\
\hline \multicolumn{3}{|l|}{$\begin{array}{l}\text { Les caractéristiques structurales peuvent } \\
\text { inclure: }\end{array}$} \\
\hline Microcéphalie à la naissance & $\begin{array}{l}\text { Oui, centile non } \\
\text { précisé }\end{array}$ & Oui, $\leq$ au $3^{\mathrm{e}}$ percentile \\
\hline Anomalies structurales & Oui & Oui \\
\hline Troubles neurologiques graves & Oui & Oui \\
\hline Troubles neurologiques légers & Oui & Non \\
\hline
\end{tabular}

Cet article a fait l'objet d'un examen par les pairs.

De l'Hôpital pour enfants, Centre des sciences de la santé, départements de pédiatrie et de santé de l'enfant, de biochimie et de génétique médicale, Université du Manitoba, Winnipeg (Man.) (Chudley); du Department of Educational and Counselling Psychology and Special Education, Université de la Colombie-Britannique, Asante Centre for Fetal Alcohol Syndrome, Maple Ridge (C.-B.) (Conry); du département d'obstétrique et de gynécologie, Université d'Ottawa, Ottawa (Ont.) (Cook); du Department of Pediatrics, Université de la Colombie-Britannique, C.-B. Children's Hospital, Vancouver (C.-B.) (Loock); du Provincial Medical Genetics Program, St. John's (T.N.-L.); du Department of Pediatrics, Université Memorial de Terre-Neuve, Saint John's (T.-N.-L.) (Rosales); du département de pédiatrie, Hôpital Georges-Dumont, Moncton (N.-B.) (Leblanc).

Intérêts concurrents : Aucun déclaré.

Contributions : Tous les auteurs ont contribué également à ce manuscrit. Tous les auteurs ont apporté une contribution substantielle à la conception du document ou à l'acquisition des données ou à l'analyse et l'interprétation des données, et ont rédigé la version préliminaire de l'article ou ont fait la révision critique d'un important contenu intellectuel. Ils ont donné leur approbation finale à la version présentée pour publication.

Remerciements : Le présent travail a pu être mené à bien grâce aux équipes de l'ETCAF de l'Agence de santé publique du Canada et 
de la Direction générale de la santé des Premières nations et des Inuits de Santé Canada. Les auteurs tiennent à remercier les nombreux cliniciens et autres personnes qui ont aidé à élaborer les lignes directrices, les ont examinées et ont fourni une rétroaction, particulièrement les membres du Comité consultatif national sur l'ETCAF de l'Agence de santé publique du Canada, les $\mathrm{D}^{\text {rs }}$ Fred Boland, Susan Astley et Sterling Clarren, et le groupe de travail scientifique sur le diagnostic des Centers for Disease Control and Prevention. La présente recherche a été financée par l'Agence de santé publique du Canada et par la Direction générale de la santé des Premières nations et des Inuits de Santé Canada.

\section{Références}

1. Jones KL, Smith DW. Recognition of the fetal alcohol syndrome in early infancy. Lancet 1973;2(7836):999-1001.

2. Sokol RJ, Delaney-Black V, Nordstrom B. Fetal alcohol spectrum disorder. 7AMA 2003;290(22):2996-9.

3. Streissguth AP. Fetal alcohol syndrome: a guide for families and communities. Baltimore : Paul H. Brookes; 1997.

4. Stratton K, Howe C, Battaglia FC. Fetal alcohol syndrome: diagnosis, epidemiology, prevention, and treatment. Washington : Institute of Medicine and National Academy Press; 1996. Disponible : www.nap.edu/books/0309052920/html/index.html (consulté le 1er septembre 2004).

5. Streissguth AP. Maternal drinking and the outcome of pregnancy: implications for child mental health. Am f Orthopsychiatry 1977;47(3):422-31.

6. Déclaration conjointe : Prévention du syndrome d'alcoolisme foetal (SAF) et des effets de l'alcool sur le fotus (EAF) au Canada. Ottawa : Santé Canada; 1996.

7. Roberts G., Nanson J. Meilleures pratiques - Syndrome d'alcoolisme fotal/effets de l'alcool sur le fotus et les effets des autres drogues pendant la grossesse. Ottawa : Santé Canada; 2003.

8. Chavez GF, Cordero JF, Becerra JE. Leading major congenital malformations among minority groups in the United States, 1981-1986. MMWR 1988;37(3):17-24

9. Sokol RJ, Clarren SK. Guidelines for use of terminology describing the impact of prenatal alcohol on the offspring. Alcohol Clin Exp Res 1989;13(4):597-8.

10. Sampson PD, Bookstein FL, Barr HM, Streissguth AP. Prenatal alcohol exposure, birthweight, and measures of child size from birth to age 14 years. $\mathrm{Am}$ 7 Public Health 1994;84(9):1421-8.

11. Sampson PD, Streissguth AP, Bookstein FL, Little RE, Clarren SK, Dehaene $\mathrm{P}$, et al. Incidence of fetal alcohol syndrome and prevalence of alcohol-related neurodevelopmental disorder. Teratology 1997;56(5):317-26.

12. Robinson GC, Conry JL, Conry RF. Clinical profile and prevalence of fetal alcohol syndrome in an isolated community in British Columbia. CMA7 1987;137(3):203-7.

13. Williams RJ, Odaibo FS, McGee JM. Incidence of fetal alcohol syndrome in northeastern Manitoba. Can 7 Public Health 1999;90(3):192-4.

14. Square D. Fetal alcohol syndrome epidemic on Manitoba reserve. CMAf 1997; 157(1):59-60.

15. Asante KO, Nelms-Maztke J. Report on the survey of children with chronic handicaps and fetal alcohol syndrome in the Yukon and Northwest British Columbia. Whitehorse : Council for Yukon Indians; 1985.

16. Habbick BF, Nanson JL, Snyder RE, Casey RE, Schulman AL. Foetal alcohol syndrome in Saskatchewan: unchanged incidence in a 20-year period. Can 7 Pub Health 1996;87(3):204-7.

17. Sood B, Delaney-Black V, Covington C, Nordstrom-Klee B, Ager J, Templin T, et al. Prenatal alcohol exposure and childhood behavior at age 6 to 7 years: I. dose-response effect. Pediatrics 2001;108(2):E34.

18. Bingol N, Schuster C, Fuchs M, Iosub S, Turner G, Stone RK, et al. The influence of socioeconomic factors on the occurrence of fetal alcohol syndrome. Adv Alcohol Subst Abuse 1987;6(4):105-18.

19. Astley SJ, Bailey D, Talbot C, Clarren SK. Fetal alcohol syndrome (FAS) primary prevention through FAS diagnosis: I. Identification of high-risk birth mothers through the diagnosis of their children. Alcohol Alcohol 2000;35(5):499-508.

20. Jacobson, JL, Jacobson, SW. Prenatal alcohol exposure and neurobehavioral development: Where is the threshold? Alcobol Health Res World 1994;18:30-6.

21. Jacobson JL, Jacobson SW. Drinking moderately and pregnancy. Effects on child development. Alcohol Res Health 1999;23(1):25-30.

22. Streissguth A, Barr H, Kogan J, Bookstein F. Primary and secondary disabilities in fetal alcohol syndrome. Dans : Streissguth AP, Kanter J. editors. The challenge of fetal alcohol syndrome: overcoming secondary disabilities. Seattle : University of Washington Press; 1997:25-39.

23. Astley SJ, Bailey D, Talbot T, Clarren SK. Fetal alcohol syndrome (FAS) primary prevention through FAS diagnosis: II. A comprehensive profile of 80 birth mothers of children with FAS. Alcohol Alcobol 2000;35(5):509-19.

24. Chudley AE, Longstaffe SE. Fetal alcohol syndrome and fetal alcohol spectrum disorder. Dans : Cassidy S, Allanson J. editors. Management of genetic syndromes (2e éd.) New York : John Wiley and Sons; 2004.

25. Astley SJ, Clarren SK. Diagnostic guide for fetal alcohol syndrome and related conditions: the 4-Digit Diagnostic Code (2e éd.). Seattle : University of Washington Publication Services; 1999.
26. Clarke ME, Tough SC. A national survey regarding knowledge and attitudes of health professionals about fetal alcohol syndrome. Ottawa : Santé Canada; 2003.

27. Lemoine P, Harousseau H, Borteyru JP, Menuet JC. Les enfants de parents alcooliques - anomalies observées : à propos de 127 cas. Ouest Med 1968;21:476-82.

28. Jones KL, Smith DW, Ulleland CN, Streissguth AP. Pattern of malformation in offspring of chronic alcoholic mothers. Lancet 1973;1(7815):1267-71.

29. Clarren SK, Smith DW. The fetal alcohol syndrome. Lamp 1978;35(10):4-7.

30. Astley SJ. Clarren SK. Diagnosing the full spectrum of fetal alcohol-exposed individuals: introducing the 4-Digit Diagnostic Code. Alcobol Alcobol 2000;35(4):400-10.

31. Benoit T, Bowes MD, Bowman N, Cantin D, Chudley A, Crolly D, et al Telemedicine diagnosis for fetal alcohol syndrome - the Manitoba experience. Pediatr Child Health 2002;7:147-51.

32. Astley SJ, Stachowiak J, Clarren SK, Clausen C. Application of the fetal alcohol syndrome facial photographic screening tool in a foster care population. $\mathcal{F}$ Pediatr 2002;141(5):712-7.

33. Astley SJ, Clarren SK. Measuring the facial phenotype of individuals with prenatal alcohol exposure: correlations with brain dysfunction. Alcohol Alcohol 2001;36(2):147-59.

34. Hennessy RJ, Kinsella A, Waddington JL. 3D laser surface scanning and geometric morphometric analysis of craniofacial shape as an index of cerebrocraniofacial morphogenesis: initial application to sexual dimorphism. Biol Psychiatry 2002;51(6):507-14.

35. Da Silveira AC, Daw JL Jr, Kusnoto B, Evans C, Cohen M. Craniofacial applications of three-dimensional laser surface scanning. 7 Craniofac Surg 2003;14(4);449-56.

36. Secretary of Health and Human Services. 10th special report to the US Congress on alcohol and health: highlights from current research. Washington : U.S Department of Health and Human Services, Public Health Service, National Institutes of Health, National Institute on Alcohol Abuse and Alcoholism; 2000.

37. Bradley KA, Boyd-Wickizer J, Powell SH, Burman ML. Alcohol screening questionnaires in women: a critical review. FAMA 1998;280(2):166-71.

38. Russell M, Martier SS, Sokol RJ, Mudar P, Bottoms S, Jacobson S, et al. Screening for pregnancy risk-drinking. Alcohol Clin Exp Res 1994;18(5):1156-61.

39. Astley SJ, Clarren SK. A case definition and photographic screening tool for the facial phenotype of fetal alcohol syndrome. 7 Pediatr 1996;129(1):33-41.

40. Jones KL, Smith DW. The fetal alcohol syndrome. Teratology 1975;12(1):1-10

41. Thomas IT, Gaitantzis YA, Frias JL. Palpebral fissure length from 29 weeks gestation to 14 years. 7 Pediatr 1987;111:267-8.

42. Hall JG, Froster-Iskenius UG, Allanson JE (sous la direction de). Handbook of normal physical measurements. Oxford : Oxford University Press; 1989:149-50.

43. Farkas LG. Anthropometry of the head and face (2e éd.). New York : Raven Press; 1994

44. Moore ES, Ward RE, Jamison PL, Morris CA, Bader PI, Hall BD. New perspectives on the face in fetal alcohol syndrome: what anthropometry tells us. Am 7 Med Genet 2002;109(4):249-60.

45. Mattson SN, Riley EP, Gramling L, Delis DC, Jones KL. Heavy prenatal alcohol exposure with or without physical features of fetal alcohol syndrome leads to IQ deficits. 7 Pediatr 1997;131(5):718-21.

46. Enquête nationale sur la santé de la population, 1994-1995. Ottawa : Statistique Canada; 1995. Disponible : http://www.statcan.ca/francais/concepts/nphs/in dex_f.htm (consulté le 11 septembre 2004).

47. Enquête longitudinale nationale sur les enfants et les jeunes, 1994-1995. Ottawa : Statistique Canada; 1995.

48. Enquête longitudinale nationale sur les enfants et les jeunes, 1998-1999. Ottawa : Statistique Canada; 1999

49. Automne 2002 - Sondage auprès des Premièresnations vivant dans les réserves. Ottawa : EKOS Research; 2002.

50. Stoler JM, Huntington KS, Petersen CM, Daniel P, Aboagye KK, Lieberman $\mathrm{E}$, et al. The prenatal detection of significant alcohol exposure with maternal blood markers. 7 Pediatr 1998; 133(3):346-52.

51. Moore C, Jones J, Lewis D, Buchi K. Prevalence of fatty acid ethyl esters in meconium specimens. Clin Chem 2003;49(1):133-6.

52. Bearer CF. Meconium as a biological marker of prenatal exposure. Ambul Pediatr 2003;3(1):40-3

53. Chan D, Bar-Oz B, Pellerin B, Paciorek C, Klein J, Kapur B, et al. Population baseline of meconium fatty acid ethyl esters among infants of non-drinking women in Jerusalem and Toronto. Ther Drug Monit 2003;25(3):271-8.

54. National Center on Birth Defects and Developmental Disabilities Centers for Disease Control and Prevention Department of Health and Human Services. Fetal Alcohol Syndrome: Guidelines for Referral and Diagnosis: National Task Force on Fetal Alcohol Syndrome and Fetal Alcohol Effect; 2004 http://www.cdc.gov/ncbddd/fas/documents/FAS_guidelines_accessible.pdf

Correspondance : $D^{r}$ Jocelynn L. Cook, équipe ETCAF, Agence de santé publique du Canada, Division de l'enfance et de I'adolescence, Immeuble Jeanne-Mance, ge étage, Pré Tunney, Localisateur postal 1909C2, Ottawa (Ont.) K1A OK9; jocelynn@primus.ca 


\begin{tabular}{|c|c|c|c|c|}
\hline Participant & Ville & Province & Profession & $\begin{array}{l}\mathrm{N}^{\text {bre }} \text { de } \\
\text { consultations }\end{array}$ \\
\hline Albert Chudley & Winnipeg & Man. & $\begin{array}{l}\text { Médecin, Clinic for Drug and Alcohol Exposed } \\
\text { Children }\end{array}$ & 12 \\
\hline Al Kircher & Winnipeg & Man. & Psychologue & 1 \\
\hline Andrea Moser & Ottawa & Ont. & Service correctionnel du Canada & 1 \\
\hline Anne Fuller & Vancouver & C.-B. & $\begin{array}{l}\text { BC Ministry of Children and Family } \\
\text { Development }\end{array}$ & 1 \\
\hline Annette Lemire & Edmonton & Alb. & Santé et Mieux-être & 2 \\
\hline Arthur Blue & Brandon & Man. & Native Psychologists in Canada & 1 \\
\hline Ben Gifford & Calgary & Alb. & Médecin, Alberta Children's Hospital & 1 \\
\hline Billie Jean Benisty & Ottawa & Ont. & Santé Canada & 2 \\
\hline Bob Armstrong & Vancouver & C.-B. & $\begin{array}{l}\text { Médecin, BC Women's and Children's } \\
\text { Hospital }\end{array}$ & 2 \\
\hline Bonnie Baxter & Vancouver & C.-B. & Orthophoniste & 1 \\
\hline Brad Bell & Whitehorse & Yukon & Santé et Mieux-être & 1 \\
\hline Brian Marder & Edmonton & Alb. & Orienteur professionnel & 1 \\
\hline Bryce Lark & Whitehorse & Yukon & Santé et Mieux-être & 1 \\
\hline Carol Gregson & Iqualuit & NT & Nunavut Department of Health & 1 \\
\hline Carol Woodworth & Vancouver & C.-B. & Orthophoniste, Asante Centre for FAS & 1 \\
\hline Cathie Royle & St. John's & T.-N.-L. & $\begin{array}{l}\text { Child Youth and Family Programs, NL Dept } \\
\text { Health and Community Services }\end{array}$ & 2 \\
\hline Christine Lilley & Vancouver & C.-B. & $\begin{array}{l}\text { Psychologue, BC Women's and Children's } \\
\text { Hospital }\end{array}$ & 1 \\
\hline Christine Loock & Vancouver & C.-B. & $\begin{array}{l}\text { Médecin, BC Women's and Children's } \\
\text { Hospital }\end{array}$ & 11 \\
\hline Claudette Landry & Fredericton & N.-B. & $\begin{array}{l}\text { Santé publique, ministère de la Santé et du } \\
\text { Mieux-être du Nouveau-Brunswick }\end{array}$ & 2 \\
\hline Dan Dubovsky & Washington & DC & Spécialiste du SAF, FAS Center of Excellence & 2 \\
\hline Darlene MacDonald & Ottawa & Ont. & Santé Canada & 2 \\
\hline Darren Joslin & Edmonton & Alb. & Santé et Mieux-être & 2 \\
\hline Dawn Ridd & Winnipeg & Man. & Santé Manitoba & 1 \\
\hline Del Nyberg & & C.-B. & BC Health & 2 \\
\hline Diane Fast & Vancouver & C.-B. & $\begin{array}{l}\text { Psychiatre, , BC Women's and Children's } \\
\text { Hospital }\end{array}$ & 1 \\
\hline Donna Ludvigsen & Edmonton & Alb. & Santé et Mieux-être & 1 \\
\hline Edward Cross & Kahnawake & Qué. & Spécialiste en éducation & 2 \\
\hline Elaine Orrbine & Ottawa & Ont. & $\begin{array}{l}\text { Association canadienne des centres de santé } \\
\text { pédiatriques, les chaires de recherche } \\
\text { canadiennes en pédiatrie }\end{array}$ & 1 \\
\hline Ellen Fantus & Toronto & Ont. & $\begin{array}{l}\text { Psychologue, Toronto Hospital for Sick } \\
\text { Children }\end{array}$ & 1 \\
\hline Faye Brooks & Ottawa & Ont. & $\begin{array}{l}\text { Association des infirmières et infirmiers du } \\
\text { Canada }\end{array}$ & 1 \\
\hline Faye Stark & Fort Providence & T. du N.-O. & Santé et Mieux-être & 1 \\
\hline $\begin{array}{l}\text { Fjola Hart- } \\
\text { Wasekeeiskaw }\end{array}$ & Ottawa & Ont. & $\begin{array}{l}\text { Association des infirmières et infirmiers } \\
\text { autochtones du Canada }\end{array}$ & 2 \\
\hline Fred Boland & Kingston & Ont. & Psychologue, Queen's University & 6 \\
\hline
\end{tabular}




\begin{tabular}{|c|c|c|c|c|}
\hline \multicolumn{5}{|l|}{ Annexe 1 : suite } \\
\hline Gail Andrew & Edmonton & Alb. & Médecin, Glenrose Rehabilitation Hospital & 4 \\
\hline Gideon Koren & Toronto & Ont. & Médecin, Toronto Hospital for Sick Children & 6 \\
\hline Graham Robinson & Ottawa & Ont. & GRC & 1 \\
\hline Guy Burbon & Ottawa & Ont. & Solliciteur général Canada & 1 \\
\hline Hasu Rajani & Cold Lake & Alb. & Médecin, Lakeland Centre for FAS & 2 \\
\hline Holly Mackay & Ottawa & Ont. & Santé Canada & 1 \\
\hline Irena Nulman & Toronto & Ont. & Médecin, Toronto Hospital for Sick Children & 3 \\
\hline Jacquelyn Bertrand & Atlanta & GA & $\begin{array}{l}\text { Psychologue, Centers for Disease Control } \\
\text { and Prevention }\end{array}$ & 1 \\
\hline Jan Lutke & Vancouver & C.-B. & BC FAS Support Network & 1 \\
\hline Janice Birney & Ottawa & Ont. & Affaires indiennes et du Nord Canada & 1 \\
\hline Jasjeet Sidhu & Atlanta & GA & Médecin, CDC & 1 \\
\hline Jo Nanson & Saskatoon & Sask. & Psychologue & 4 \\
\hline Joanne Rovet & Toronto & Ont. & $\begin{array}{l}\text { Psychologue, Toronto Hospital for Sick } \\
\text { Children }\end{array}$ & 2 \\
\hline Joanne Weinberg & Vancouver & C.-B. & Neuroscientifique & 1 \\
\hline Jocelynn Cook & Ottawa & Ont. & Santé Canada & 11 \\
\hline Jocyline Gauthier & Whitehorse & Yukon & Santé et Mieux-être & \\
\hline John Arnett & Winnipeg & Man. & Psychologue & 1 \\
\hline John Godel & Campbell River & C.-B. & Médecin & 1 \\
\hline John Service & Ottawa & Ont. & Société canadienne de psychologie & 1 \\
\hline Julie Conry & Vancouver & C.-B. & Psychologue, Asante Centre for FAS & 11 \\
\hline Karen Archbell & Toronto & On. & Ministère de la santé de l'Ontario & 1 \\
\hline Kathleen Montpetit & Montreal & Qué. & Ergothérapeute, Hôpital Shriner & 2 \\
\hline Kathy Horne & Edmonton & Alb. & Psychologue, Glenrose Rehabilitation Hospital & \\
\hline Kathy Jones & Winnipeg & Man. & $\begin{array}{l}\text { Psychologue, West Region First Nation } \\
\text { Child and Family Centre }\end{array}$ & 1 \\
\hline Kelly Stone & Ottawa & Ont. & Directeur, Santé Canada & 3 \\
\hline Kwadwo Asante & Vancouver & C.-B. & Médecin, Asante Centre for FAS & 4 \\
\hline Leigh Wincott & Thompson & Man. & Médecin, Thompson Diagnostic Clinic for FAS & 2 \\
\hline Leslie Grob & Regina & Sask. & SK Health & 2 \\
\hline Margaret Clarke & Calgary & Alb. & Médecin, Alberta Children's Hospital & 3 \\
\hline Marie Adele Davis & Ottawa & Ont. & Société canadienne de pédiatrie & 1 \\
\hline Marilou Reeve & Ottawa & Ont. & Justice pour les jeunes & 1 \\
\hline Marilyn Van Bibber & Vancouver & C.-B. & BC FAS Resource Network & 1 \\
\hline Mary Cox-Millar & Winnipeg & Man. & $\begin{array}{l}\text { Coordonnatrice, Clinic for Drug and Alcohol } \\
\text { Exposed Children }\end{array}$ & 1 \\
\hline Mary Ellen Baldwin & Calgary & Alb. & Psychologue, Alberta Children's Hospital & 1 \\
\hline Mary Johnston & Ottawa & Ont. & Santé Canada & 4 \\
\hline Mary Lynch & Saint John & N.-B. & $\begin{array}{l}\text { Ministère des services familiaux du Nouveau- } \\
\text { Brunswick }\end{array}$ & 1 \\
\hline Mercedes Mompel & Toronto & Ont. & Santé et Mieux-être & 1 \\
\hline
\end{tabular}




\begin{tabular}{|c|c|c|c|c|}
\hline \multicolumn{5}{|l|}{ Annexe 1 : suite } \\
\hline Michelle Dubik & Winnipeg & Man. & Enfants en santé Manitoba & 2 \\
\hline Nadine Huggins & Ottawa & Ont. & Santé Canada & 3 \\
\hline Nancy Taylor & Halifax & N.-É. & & 1 \\
\hline Nicole Chatel & Yellowknife & T. du N.-O. & Stanton Territorial Health Authority & 1 \\
\hline Nicole LeBlanc & Moncton & N.-B. & Médecin, Hôpital Georges Dumont & 5 \\
\hline Nikki Bansil & Ottawa & Ont. & Association médicale canadienne & 1 \\
\hline Pamela Massad & Ottawa & Ont. & Santé Canada & 1 \\
\hline Patricia Blakely & Saskatoon & Sask. & Médecin, Kinsmen Children's Centre & 4 \\
\hline Patricia MacPherson & Montague & I.-P.-E. & $\begin{array}{l}\text { Centre de recherche, Service correctionnel du } \\
\text { Canada }\end{array}$ & 1 \\
\hline Pearl Park & Calgary & Alb. & Orthophoniste, Alberta Children's Hospital & 1 \\
\hline Peter Waas & LaCombe & Alb. & Psychologue & 3 \\
\hline Rachelle Deneault & Whitehorse & Yukon & & 1 \\
\hline Richard Snyder & Saskatoon & Sask. & Médecin, Kinsmen Children's Centre & 1 \\
\hline Roxana Vernescu & St John's & T.-N.-L. & Psychologue, Memorial University & 1 \\
\hline Samantha Nadjiwan & Ottawa & Ont. & $\begin{array}{l}\text { First Nations Child and Family Caring Society } \\
\text { of Canada }\end{array}$ & 1 \\
\hline Sandy Clarren & Seattle & WA & Psychologue, University of Washington & 1 \\
\hline Sandy Steinwender & Iqualuit & Nunavut & Santé et Services sociaux & 1 \\
\hline Sharon Bartholomew & Ottawa & Ont. & Santé Canada & 2 \\
\hline Soo-Hong Uh & Vancouver & C.-B. & Scientifique, BC Vital Statistics & 2 \\
\hline Sterling Clarren & Seattle & WA & Médecin, University of Washington & 1 \\
\hline Suzanne Guay & Ottawa & Ont. & $\begin{array}{l}\text { Commission nationale des libérations } \\
\text { conditionnelles }\end{array}$ & 1 \\
\hline Ted Rosales & St. John's & T.-N.-L. & Médecin, Memorial University & 12 \\
\hline Terry Benoit & Winnipeg & Man. & $\begin{array}{l}\text { Médecin, Clinic for Drug and Alcohol Exposed } \\
\text { Children }\end{array}$ & 1 \\
\hline Tim Oberlander & Vancouver & C.-B. & $\begin{array}{l}\text { Médecin, BC Women's and Children's } \\
\text { Hospital }\end{array}$ & 1 \\
\hline Val Massey & Edmonton & Alb. & Psychologue, DV Massey and Associates & 1 \\
\hline Valerie Flynn & Ottawa & Ont. & Santé Canada & 2 \\
\hline Vyta Senikas & Ottawa & Ont. & $\begin{array}{l}\text { Société des obstétriciens et gynécologues du } \\
\text { Canada }\end{array}$ & 1 \\
\hline $\begin{array}{l}\text { Wendy Sky } \\
\text { Delaronde }\end{array}$ & Kahnawake & Qué. & Infirmière & 1 \\
\hline Yaya deAndrade & Vancouver & C.-B. & $\begin{array}{l}\text { Psychologue, BC Women's and Children's } \\
\text { Hospital }\end{array}$ & 1 \\
\hline Yeshodara Naidoo & Ottawa & Ont. & Santé Canada & 1 \\
\hline
\end{tabular}


Annexe 2 : Guides de mesure de la longueur de la fente palpébrale.

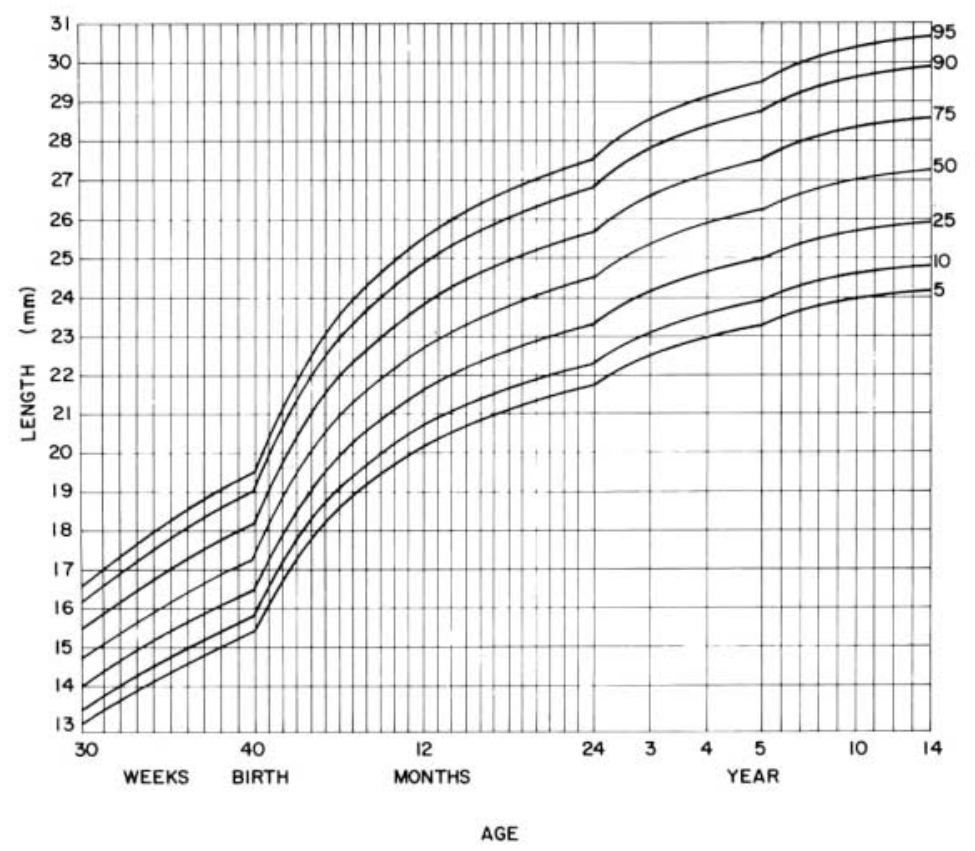

Annexe 2-1. Relation entre la longueur de la fente palpébrale et l'âge chez les enfants blancs américains des deux sexes, de la $29^{\mathrm{e}}$ semaine de grossesse à 14 ans $^{41}$.

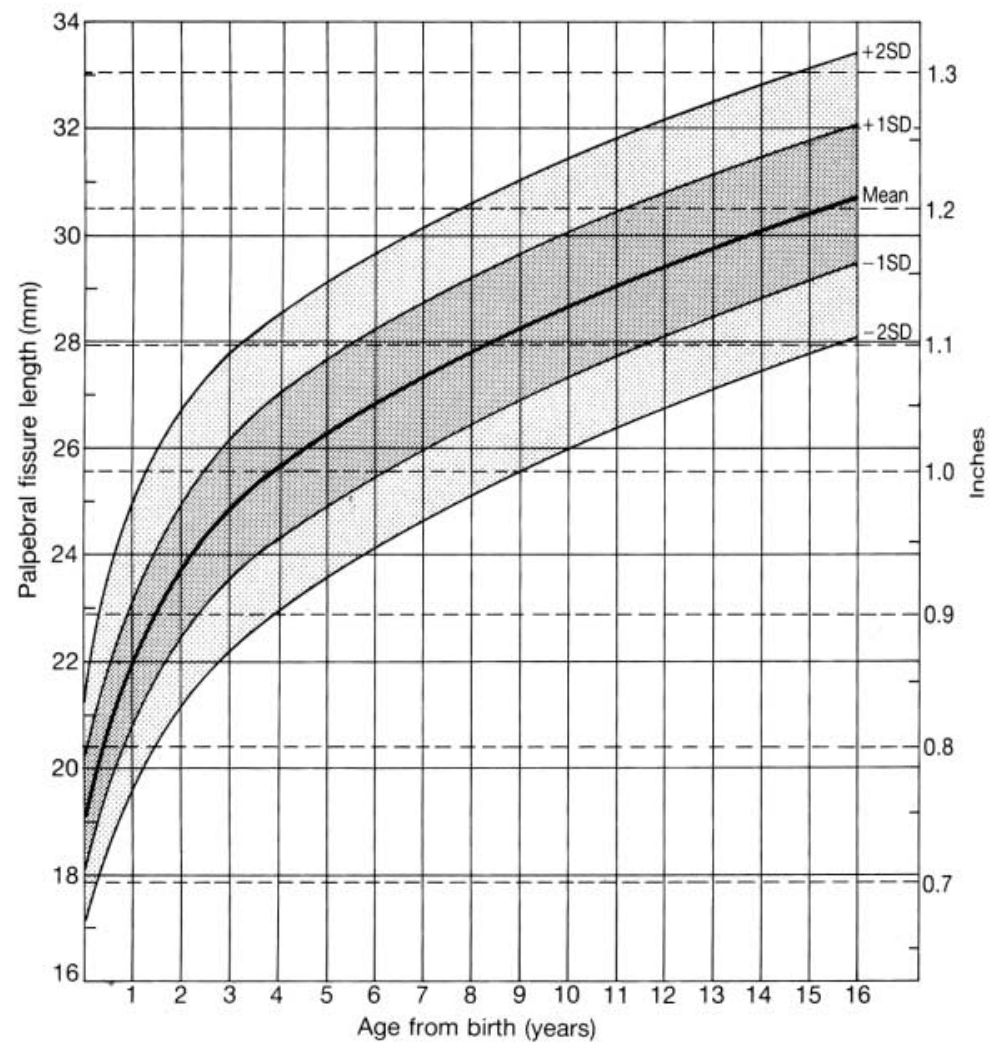

Annexe 2-2. Longueur de la fente palpébrale, pour les deux sexes, de la naissance à 16 ans $^{42}$. 


\section{Annexe 3 : Exemples des tests les plus couramment utilisés pour évaluer les domaines.}

* Des psychologues, des orthophonistes et des ergothérapeutes ont été consultés afin de savoir quels tests ils utilisaient le plus couramment. Les évaluations du fonctionnement du cerveau sont mises à jour régulièrement et il convient d'utiliser les versions les plus récentes dans la mesure du possible.

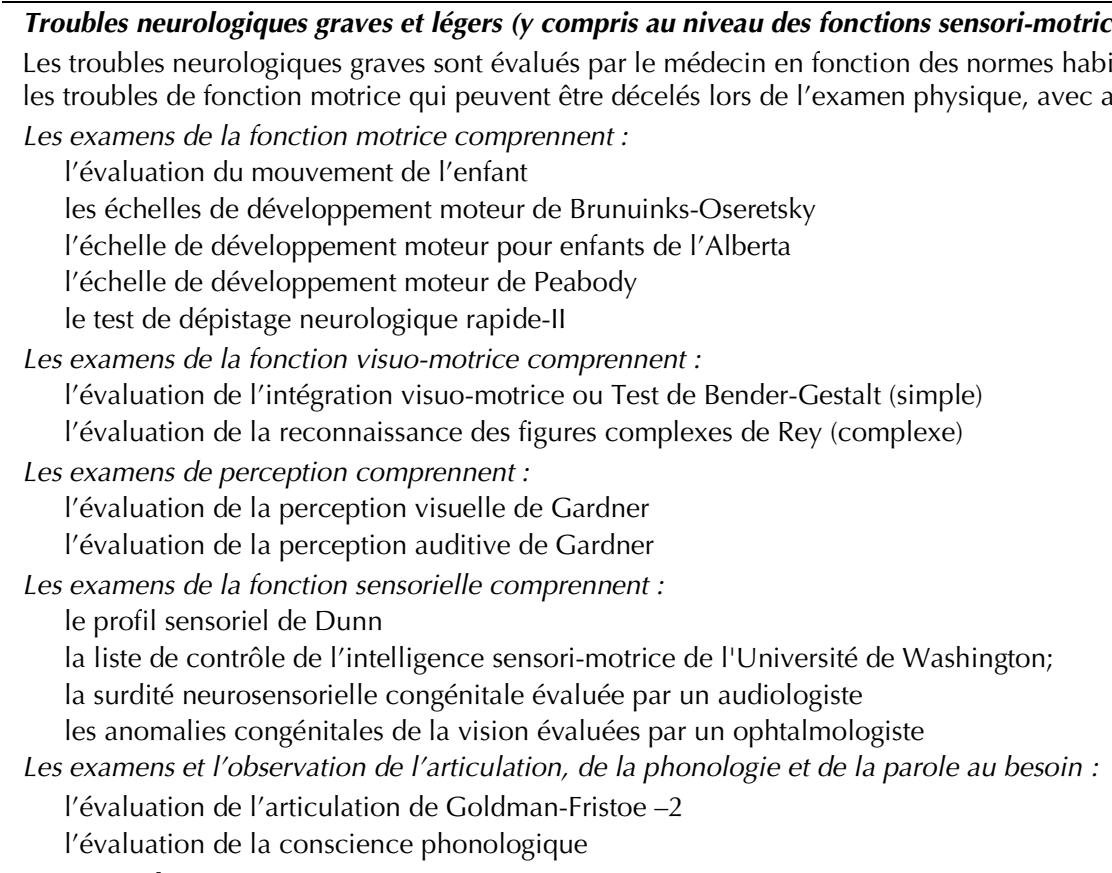

\section{Structure du cerveau}

Mesures documentées de la circonférence de la tête (circonférence occipitofrontale, inférieur au $3^{\text {e }}$ percentile) ajustées en fonction de l'âge et du sexe (lors d'un examen physique à tout âge, y compris la circonférence de la tête à la naissance) et autres preuves de dysfonction du SNC fonctionnelles ou structurales fondées sur un examen neurologique ou des techniques d'imagerie (photobalayage tomographique informatisé, imagerie par résonance magnétique, électroencéphalogramme). Les troubles neurologiques peuvent inclure les convulsions qui ne sont pas dues à un accident postnatal ou les autres signes tels qu'une capacité motrice réduite, la surdité neurosensorielle, la perte de mémoire et une mauvaise coordination œil-main.

\section{Cognition}

Les tests du fonctionnement intellectuel comprennent :

I'échelle d'intelligence de Wechsler pour enfants-III (I'utilité du test WISC-IV pour la population atteinte de l'ETCAF n'a pas encore été testée) l'échelle d'intelligence Stanford-Binet, quatrième édition (I'utilité du SB5 pour la population atteinte de l'ETCAF n'a pas encore été testée)

l'échelle d'intelligence préscolaire et primaire de Wechsler pour enfants-III

l'échelle d'habiletés différentielles

les échelles Bayley de développement des nourrissons

\section{Communication}

Les batteries de tests pour les fonctions du langage combinent habituellement les fonctions tant réceptives qu'expressives du langage, ainsi que les mots uniques et les fonctions complexes (phrases et paragraphes). Il convient de faire la distinction entre les réponses suscitées et les aptitudes de reconnaissance (choix multiples).

évaluation du vocabulaire en images de Peabody-III

évaluation du vocabulaire expressif

échelle de langage préscolaire (3 ou 4)

échelle du développement du langage de Reynell

test de compréhension auditive du langage-3

test des jetons

évaluation de la capacité d'attention

évaluation de la connaissance des mots

évaluation clinique des bases du langage (préscolaire, CEFL-3, CELF-4)

Ces mesures sont complétées par une analyse d'un échantillon du langage incluant : la longueur des énoncés, I'utilisation de phrases complexes et le repérage de mots.

Observation du langage social

Capacité narrative (PLS-E story retell); Renfrew Bus Story, Frog Where are You

(Nota : I'aspect pragmatique du langage est pris en considération dans le domaine des aptitudes sociales et adaptatives.) 


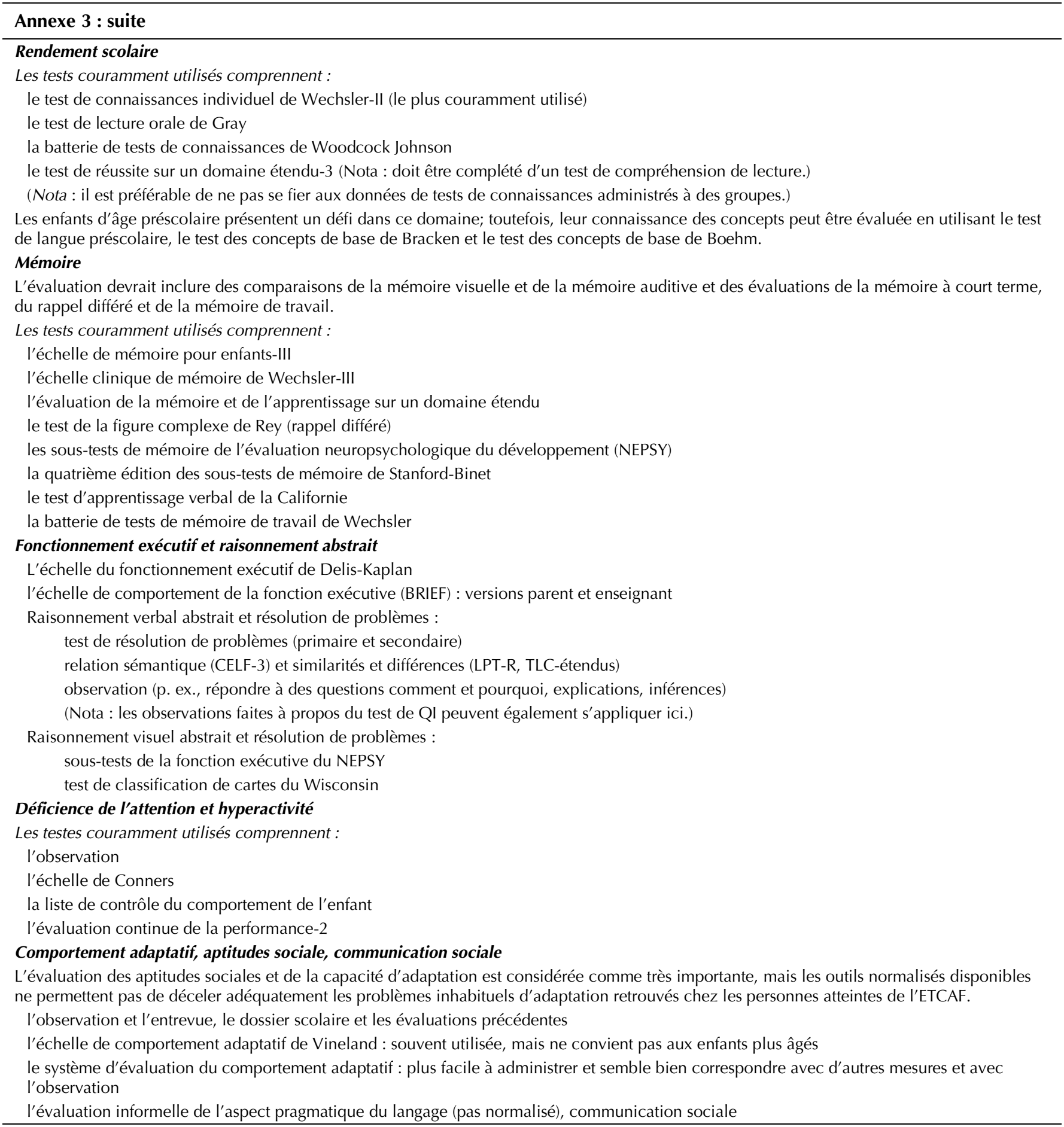

